This is the accepted version of the article:

Zamani R.R., Arbiol J.. Understanding semiconductor nanostructures via advanced electron microscopy and spectroscopy. Nanotechnology, (2019). 30. 262001: 10.1088/1361-6528/ab0b0a.

Available at: https://dx.doi.org/10.1088/1361-6528/ab0b0a 


\title{
Understanding semiconductor nanostructures via advanced electron microscopy and spectroscopy
}

\author{
Authors: Reza R. Zamani, ${ }^{1,}{ }^{*}$ Jordi Arbiol ${ }^{2,3}$ \\ ${ }^{1}$ Department of Physics, Chalmers University of Technology, Gothenburg 41296, Sweden \\ ${ }^{2}$ Catalan Institute of Nanoscience and Nanotechnology (ICN2), CSIC and BIST, Campus UAB, \\ Bellaterra, Barcelona 08193, Catalonia, Spain \\ ${ }^{3}$ ICREA, Pg. Lluís Companys 23, Barcelona 08010, Catalonia, Spain \\ Email: reza.zamani@chalmers.se; reza.r.zamani@gmail.com
}

Keywords: TEM, STEM, EELS, EDX, CL, imaging, spectroscopy, aberration-corrected scanning transmission microscopy, semiconductor nanowire

\section{Abstract}

Transmission electron microscopy (TEM) offers an ample range of complementary techniques which are able to provide essential information about the physical, chemical and structural properties of the materials at the atomic scale, and make a vast impact on our understanding of materials science, especially in the field of semiconductor 1-dimensional (1D) nanostructures. Recent advancements in TEM instrumentation, in particular aberration correction and monochromation, have enabled pioneering experiments in complex nanostructure material systems.

This Review aims to address these understandings through the applications of the methodology for semiconductor nanostructures. It points up various electron microscopy techniques, in particular scanning TEM (STEM) imaging and spectroscopy techniques, with their already-employed or potential applications on 1D nanostructured semiconductors. We keep the main focus of the paper on the electronic and optoelectronic properties of such semiconductors, and avoid expanding it further.

In the first part of the paper, we give a brief introduction to each of the STEM-based techniques, without detailed elaboration, and mention the recent technological and conceptual developments which lead to novel characterization methodologies. For further reading, we refer the audience to a handful of papers in the literature.

In the second part, we highlight the recent examples of application of the STEM methodology on the 1D nanostructure semiconductor materials, especially III-V, II-V, and group IV bare and heterostructure systems. The aim is to address the research questions on various physical properties and introduce solutions by choosing the appropriate technique that can answer the questions. Potential applications will also be discussed, the ones that have already been used for bulk and 2D materials, and have shown great potential and promise for 1D nanostructure semiconductors. 


\section{Table of contents}

1. Introduction

2. STEM technique advancements

2.1. STEM imaging techniques

2.2. Nanobeam electron diffraction - NBED

2.3. X-ray energy dispersive spectroscopy - EDX

2.4. Electron energy-loss spectroscopy - EELS

2.5. Cathodoluminescence - $\mathrm{CL}$

3. STEM applications on 1D nanostructured semiconductors

3.1. Structural properties

\subsubsection{Polarity}

3.1.2. Growth mechanisms

3.1.3. Structural defects

3.1.4. Atomic ordering

3.1.5. Quantum structures

3.1.6. Heterojunctions

3.1.7. Lattice strain

3.2. Physical properties

3.2.1. Optical properties by EELS

3.2.2. Luminescence properties via $\mathrm{CL}$

3.2.3. Electric fields via DPC imaging

4. Conclusion

5. Acknowledgements

References

\section{Introduction}

For several decades, transmission electron microscopy (TEM) has been known as a powerful method for characterization of materials $\left[{ }^{1,2}\right]$. It offers a wide range of techniques, each of which can provide various type of information about the structural, chemical, and even physical properties of the materials $\left.{ }^{3}\right]$. This has led to considerable advancements in materials science and technology. The field of semiconductors has not been an exception. It has taken advantage of this methodology to obtain essential information, expand the knowledge and utilized it to produce and improve the semiconductor devices such as light emitting diodes (LEDs) $\left[{ }^{4},{ }^{6}, 6\right]$, solar cells $\left[{ }^{7}\right]$, lasers $\left[{ }^{8},{ }^{9}\right]$, and field-effect transistors (FETs) $\left[{ }^{10}\right]$.

In the past years, the TEM technology and knowledge has been revolutionized by a few outstanding inventions that opened up new possibilities of fundamental studies on the materials. One of these inventions that has had a vast impact is the correctors of the magnetic lens aberrations, mainly the spherical aberration $\left(C_{s}\right)$, which has been commercially available since more than a decade $\left[{ }^{11},{ }^{12}\right]$. $C_{s}$ correctors are sets of multipole lenses that can compensate for the imperfections of the magnetic lenses of the microscope. This has resulted 
in a drastic improvement of the resolution [ $\left.{ }^{13}\right]$. Post-specimen $C_{s}$ correctors, known as image correctors, reduce the aberrations of the beam after interaction with the mater. This particularly improves the resolution of the images in TEM mode $\left[{ }^{14}\right]$. On the other hand, prespecimen $C_{s}$ correctors, probe correctors, adjust the STEM electron probe and reduce its aberrations before interacting with the specimen. This has improved the resolution of the STEM imaging down to sub-angstrom, which is essential for resolving special structural features such as polarity in semiconductors $\left[{ }^{15}, 16,17\right]$.

It should be noted that STEM resolution is predominantly determined by the size of the probe, and the electron source has an important role in it. The energy spread is one of the most important parameters of the electron source, which has been improved dramatically by Schottky and cold field emission guns. They have also increased the coherence as well as the brightness of the probe, and decreased the energy spread and the chromatic aberrations $\left(C_{c}\right)$.

The energy spread of the electron probe can be further decreased by using a beam monochromator, which is also one of the most groundbreaking inventions in (S)TEM technology. A monochromator spreads the electrons according to their energy, filters out the ones with higher or lower energies with a slit, and then refocuses them to a much more uniform beam. A monochromated beam increases the information limit of high-resolution TEM (HRTEM) images $\left[{ }^{18}\right]$, but more importantly, it increases the energy resolution for electron energy-loss spectroscopy (EELS). This topic will be covered in Section 2.

Apart from aberration correction and monochromation, several other technology advancements have facilitated (S)TEM data acquisition for newly designed experiments: improved signal detection systems, faster and more reliable electronics, more stable electronoptics and mechanics, digitalization and faster computation and acquisition systems (hardware and software), to name a few. Thanks to them, (S)TEM data enjoy more stability, less noise and stronger signals, and in one word, less ambiguity. On the other hand, the interpretation of the acquired data is facilitated by the expansion of the knowledge and better understanding of the phenomena occurring in the event of electron-matter interaction, hand in hand with the ability of simulations and numerical calculations.

Nowadays, state-of-the-art (S)TEMs are relatively accessible since hundreds of them are installed around the globe; and performing (S)TEM experiments is rather fast and inexpensive. Moreover, (S)TEM resolution, as well as reliability and reproducibility of the data is extremely high, and the knowledge to interpret the data is available.

In this Review, first we give a short description of the (S)TEM techniques of our focus: STEM imaging and spectroscopy techniques. We discuss the fundamentals of the techniques together with the information that can be obtained from them. Annular dark-field (ADF-), high-angle annular dark-field (HAADF-), bright-field (BF-) and annular bright-field (ABF-) STEM imaging techniques are discussed. We also point out differential phase contrast (DPC) imaging which can be performed by means of newly developed segmented (and pixelated) STEM detectors. Nanobeam electron diffraction (NBED) is also briefly described. Among spectroscopy techniques, we address energy dispersive X-ray spectroscopy (EDX), electron energy-loss spectroscopy (EELS), and cathodoluminescence ( $C L$ ). Most of these techniques have been extensively used, or have the potential to be used, for characterization of 1D nanostructured semiconductors.

(S)TEM methodology is in fact much wider than what we have mentioned here. However, we keep the focus of this Review on STEM methodology employed on nanostructured 
semiconductors. Other methods such as in-situ TEM $\left[{ }^{19},{ }^{20},{ }^{21}\right]$, electron holography $\left[{ }^{22},{ }^{23}\right]$, electron diffraction $\left[{ }^{24},{ }^{25},{ }^{26},{ }^{27}\right]$ and other TEM-based techniques are also interesting and useful, and have been widely used for 1D nanostructured materials. However, we keep them out of the scope of this Review.

In the second part we give an insight to the current applications of the aforementioned techniques on 1D nanostructured semiconductors, and we show how they have contributed in advancements in exploring the new areas of semiconductor science and technology. We mention some highlights of the employment of STEM techniques to provide appropriate answers to research questions that are arisen from the developments of semiconductor sciences. How STEM imaging answers the questions about the nanowire growth mechanisms? How the electronic bandgaps can be measured by EELS? How STEM-CL explains different luminescence behavior of quantum discs? We aim to review the currently published studies that have provided such answers. Moreover, we will also discuss potential applications of STEM methodology for future employment on nanostructured semiconductors.

\section{STEM technique advancements}

Conventional TEM (CTEM) techniques provide useful information about the materials. Development of STEM, in addition, expanded the obtainable information. It provided the capability of dwelling on each pixel in the image and producing image information, by using elastic scattering, and chemical information, by using inelastic scattering, simultaneously $\left[{ }^{28}\right]$. Here is a brief description of some of the most common imaging and spectroscopy techniques.

\subsection{STEM imaging techniques}

In STEM the electron optics after the specimen are quite simple. Therefore, it is relatively easy to obtain information from the transmitted electron. The only requirement is the use of appropriate detectors. Bright-field (BF) and annular dark-field (ADF) detectors have been the first ones used for imaging. The former mostly observes the diffraction contrast (depending on the angular size), where the latter collects a mixture of Bragg diffracted (lower angles) and incoherently scattered (wider angle) electrons, causing difficulties in interpreting the ADF images. This led to the development of high-angle annular dark-field (HAADF) detectors which eliminates a large part of the diffraction contrast $\left[{ }^{29}\right]$.

The intensity in HAADF images is mostly 'Z-contrast' which depends on the atomic number of the element and/or the thickness of the specimen. This can even be used for chemical characterization; if the elements consisting the specimen are known, one can assign the higher intensities to the heavier elements and lower intensities to lighter ones.

HAADF detectors, however, are not able to detect very light elements, especially in the vicinity of a heavier one. For this aim, annular bright-field (ABF) detector can be used, which has considerably increased the sensitivity of STEM imaging. ABF detector is similar to $B F$, but by having the middle point blocked, the contrast increases based on the classic hollow-cone illumination $(\mathrm{HCl})$ theory $\left[{ }^{30},{ }^{31}\right]$. With the phase contrast of the 'weak-phase object', it is even able to visualize $\mathrm{H}$ atoms directly [ ${ }^{32}$ ]. For those compounds that contain light elements such as $\mathrm{O}$ and $\mathrm{N}$, the Z-contrast intensity corresponding to them is too low to be visible in the HAADF-STEM images, in contrast to the brighter columns representing heavier atoms; therefore, ABF-STEM imaging can be utilized to solve the problem. It can be performed by using ABF detectors as schematically shown in Fig. 1(b) [ $\left.{ }^{33}\right]$, with a typical collection angle of 10-22 mrad. However, in the absence of such equipment, a conventional BF detector can be used together with the beam stopper blocking its central area. Alternatively, one can use the 
HAADF detector and decrease the camera length in a way that the collection angle is reduced to 10-22 mrad and electrons scattered with small angle land on the HAADF detector.

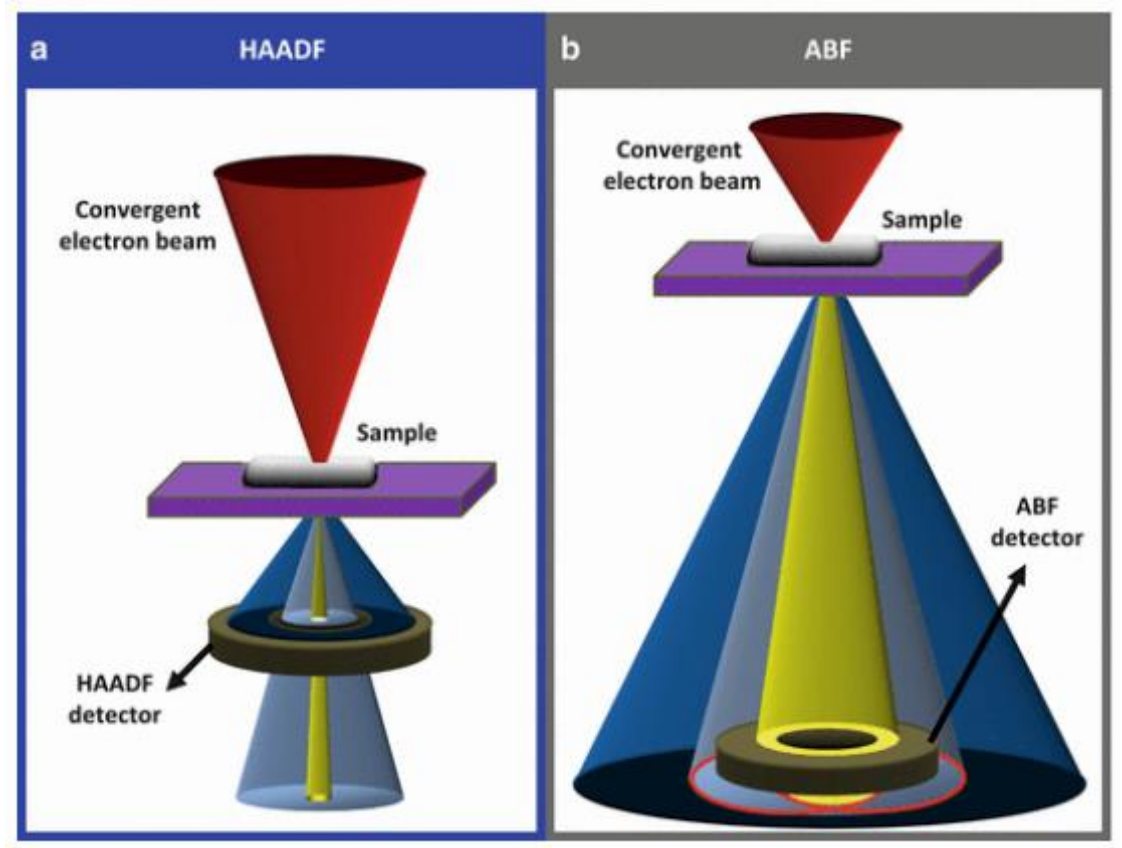

Fig. 1. Schematic illustration of (a) HAADF- and (b) ABF-STEM imaging techniques. Reprinted from [33] with the permission of Springer

More recently segmented $\left[{ }^{34}\right]$ and pixelated $\left[{ }^{35}\right]$ STEM detectors are developed. The advantage of a pixelated detector is that all the electrons can be collected at once independently of their collection angle, and thus, the output signal can be generated by selecting the desired range or even a combination of angles and sections, emulating any type of detector available. In addition, the use of segmented and/or pixelated detectors may provide information on the electric field of the material. The pixelated detectors can be used to achieve high efficiency differential phase contrast (DPC) images. They can also be utilized for ptychography $[36,37,38]$.

Segmented detectors also offer the capability of DPC imaging. Differential phase contrast reflects the gradient of the electrostatic potential of the atoms [ $\left.{ }^{39}\right]$. The basic principle of DPC imaging by segmented STEM detectors is schematically illustrated in Fig. 2. When the electron probe passes close to an atom, assumed as an isolated object, it tends toward it, causing a deflection and an asymmetry in the intensity of the signals detected by each segment of the detector. Subtracting the signals from the segments produces an antisymmetric difference profile which has a node at the atom position, and a maximum and a minimum in the immediate vicinity of it. This profile reflects the reversal of the electric field direction across the atom. By means of this method one can detect the mesoscopic polarization fields, as well as atomic-resolution electric fields. Great care is required to interpret atomic-resolution DPC data, since slight misalignment from the crystallographic orientation, thickness of the sample, or strongly scattering objects (e.g. heavier elements) can cause artefacts [39]. 

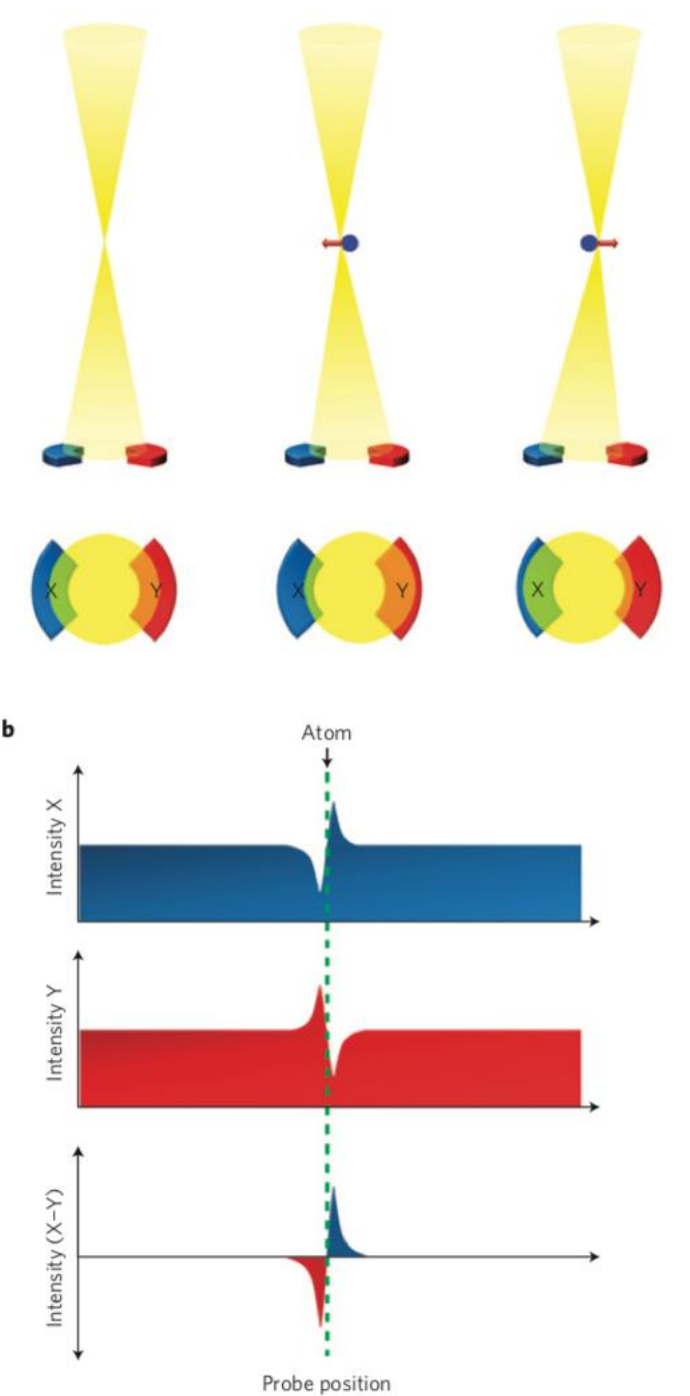

Fig. 2. Schematic illustration of DPC imaging, (a) electron trajectory in the vicinity of an atom and the way it reaches the two segments of the detector, (b) the intensity profile of the image on each detector and the subtracted intensity $X-Y$ showing the DPC intensity. Reprinted from [39] with the permission of Nature

So far, we have discussed the development of the experimental data acquisition techniques. Image simulation, on the other hand, is of essence in order to interpret and corroborate the experimental data. Interaction of the electron beam with the matter is a complicated phenomenon, especially when the channeling effects are considered $\left[{ }^{40}\right]$. In addition, hidden features can create special reflections in the 2D (S)TEM images that can be totally misleading. By image simulation we can have a better understanding about the outcome of such complex interactions. Several commercial and open-access software tools are currently available: JEMS $\left[{ }^{41}\right]$, QSTEM $\left[{ }^{42}\right]$, Dr. Probe $\left[{ }^{43}\right]$, STEM_CELL $\left[{ }^{44}\right]$, STEMSIM $\left[{ }^{45}\right]$, StatSTEM $\left[{ }^{46}\right]$, to name a few. For STEM image simulation the Kirkland's multislice approach is common $\left[{ }^{47}\right]$. They can create $3 \mathrm{D}$ atomic models of the crystal structures or import them from other software tools such as Rhodius $\left[{ }^{48}\right]$, take into account the electron probe contribution by considering the experimental parameters, and simulate STEM images.

With the development of aberration-corrected TEMs, all the aforementioned experimental imaging techniques can provide information with atomic spatial resolution. In Section 3, we 
show a handful of examples of sub-angstrom STEM imaging on nanostructured semiconductor material systems.

\subsection{Nanobeam electron diffraction - NBED}

Nanobeam electron diffraction (NBED) in STEM mode is a recently developed method which is capable of mapping nanometer scale strain with high precision of $0.1 \%$ and spatial resolution of $\sim 1 \mathrm{~nm}$. Strain analysis with such precision and spatial resolution is now possible thanks to the new developments in the TEM technology, especially development of chargecoupled device (CCD) cameras with high acquisition speed, more stability in the TEMs that hinders the mechanical drifts, and more importantly employment of aberration correctors, providing a narrow and coherent beam. To measure the strain field, HAADF-STEM images are recorded simultaneously with the NBED patterns at each pixel. Then the recorded NBED patterns are compared to a reference pattern in order to detect the displacements of the diffraction disks [ $\left.{ }^{49}\right]$.

A further step in improvement of strain analysis, is combining STEM-NBED with in-situ micromanipulation. With this approach one can measure the electrical properties, for instance by plotting the current-voltage (I-V) curve, while the mechanical force is being employed and the strain is being calculated by monitoring the displacements of the NBED disks $\left[{ }^{50,},{ }^{51}\right]$.

An alternative method for strain analysis is geometric phase analysis (GPA) $\left[{ }^{52}\right]$. In comparison to GPA, NBED-STEM is much more sensitive, and it can detect the strain that is within the error of GPA. On the other hand, GPA can have higher spatial resolutions, down to atomic scale, if it is provided with a high quality (S)TEM image. In addition, a method of quantitative STEM imaging, based on non-rigid registration and averaging of image series, can also be used for picometer-precision analysis of atomic positions $\left[{ }^{53}\right]$ and hence strain analysis $\left[{ }^{54}\right]$.

\subsection{X-ray energy dispersive spectroscopy - EDX}

$X$-ray energy dispersive spectroscopy (EDX) is one of the most common spectroscopy techniques. Characteristic X-rays produced in the event of electron-matter interaction, provide chemical information of the specimen. They are produced when electron beam ejects one of the electrons from the inner shells of the element, and another electron from an outer shell substitutes it. In a TEM, EDX detector is placed above the specimen in the objective lens pole piece gap. It must fairly close to the specimen in order to increase the collection angle and receive more of the emitted X-rays. However, there are limitations due to the geometry of the pole pieces and the available space in their gap. Besides, the detection technology and electronics play an important role in EDX data acquisition.

The traditional limitations of EDX has mainly been the small solid angle and slow count rate because of the small fraction of X-rays collected by the detector. Moreover, beam currents generated by the electron gun were less strong. Multiple silicon drift detectors (SDDs) are substituting the conventional Si ones, and together with faster and more efficient electronics, as well as the employment of Schottky field-emission guns (FEGs), they have increased the $X-$ ray collection efficiency by more than an order of magnitude $\left[{ }^{55}\right]$.

\subsection{Electron energy-loss spectroscopy - EELS}


Electron energy-loss spectroscopy (EELS) is a technique that obtains information from the inelastically scattered electrons, the ones that have interacted with the atoms and have lost some of their energy. The energy loss is characteristic of the element and is seen as an edge on a background, and can be used for chemical mapping. In comparison to EDX, EELS demands deeper knowledge for the interpretation of the data, and it requires strictly thinner specimens. However, beside higher signal collection, and absolute and standardless quantification, it has several other advantages over EDX, which will be discussed in the following.

Apart from qualitative and quantitative chemical mapping of the material, EELS can provide further information, such as electronic structure of the compounds $\left[{ }^{56}\right]$, plasmonic resonances of metallic structures $\left[{ }^{57}\right]$, bandgap of semiconductors $\left[{ }^{58}\right]$, and very recently, even performing vibrational spectroscopy, i.e. detection of material phonons, giving information about their bonding arrangement and chemical compounds [ $\left.{ }^{59}\right]$. Here we focus on determination of the bandgap and band structure by valence EELS (VEELS), since it is of high importance for semiconductor research. We discuss that monochromation of the electron probe has made a dramatic impact on the accuracy and reliability of these measurements.

In the EEL spectrum of semiconductors, as well as some insulating materials, a forbiddentransition region exists immediately after the zero-loss peak (ZLP) and before the plasmon peaks (low-loss region), in which no energy loss occurs. This is due to the absence of any permitted states in the bandgap. Therefore, the width of this region usually indicates the bandgap of the material. However, bandgap measurement is not always straightforward, as we need to take into account other low loss EELS contributions (e.g.: lower energy plasmons, phonons, and the Cerenkov radiation contribution) which may appear in the bandgap region $\left[{ }^{60}\right]$. The important experimental parameters are the full-width half-maximum (FWHM) and tenth-maximum (FWTM) of the ZLP. The former determines the energy resolution, and the latter is the tail of the ZLP which overlaps with the forbidden-transition region. Previously in non-monochromated TEMs, the tail of the ZLP extended further and covered the onset of the first plasmon peaks, resulting in relatively large errors in bandgap measurements. Monochromated electron probes, however, narrow down the energy spread which result in narrower ZLP with smaller tail, providing much higher reliability in bandgap measurement and allowing for measuring the much narrower ones. Recently, the unprecedented energy resolution (below $10 \mathrm{meV}$ ) of the monochromated STEM-dedicated microscope reported by Krivanek et al [59] has opened up the way for such measurements with high accuracy.

Apart from monochromation, improvements in spectroscopes, i.e. faster and better acquisition, higher energy dispersion, and larger range of detection, play a crucial role in EELS experiments. Such developments wedded to the ones of the monochromated electron probe have dramatically increased the accuracy and reliability of bandgap measurements.

\subsection{Cathodoluminescence - $\mathrm{CL}$}

Cathodoluminescence $(\mathrm{CL})$ is another spectroscopy technique with great potential to explore the optical properties of the semiconductors. As can be understood from the name, it is based on the 'luminescence' phenomenon, i.e. emission of photons from the material as a result of interaction with the microscope's electron beam. Luminescence occurs when the recombination of electron-hole pairs is radiative. It may involve transitions between the states in the conduction or valence bands and those within the bandgap caused by structural defects [ $\left.{ }^{61}\right]$. In the absence of deep recombination centers, most the emission shows the energy of the bandgap, since most of the recombinations arise close to the bandgap energy $\left[{ }^{62}\right]$. CL can 
be used both in scanning electron microscope (SEM) and STEM. SEM-CL is widely used since the collection of the photons is easier. STEM-CL has more difficulties in this sense, since the objective pole piece gap geometry in TEM limits the position and size of a mirror to collect the photons. A drawback of $C L$ has been its inability to resolve the luminescence properties with high spatial resolution. Recently this problem has been tackled and better spatial and spectral resolutions are achieved by improvements in the coherency and brightness of the electron probe, as well as advancements in detection systems, i.e. increasing the collection angle and minimizing the signal loss to keep the spectral resolution. Multiple mirrors for collecting the photons are also used recently [ ${ }^{63}$. The $\mathrm{CL}$ range (both for SEM- and STEM-CL), however, is still limited to UV to visible light, which is suitable for relatively wide-bandgap materials. Yet it can cover a fairly wide range of semiconductors. Another challenge with STEM-CL is the sensitivity of the method to any kind of structural defects. For instance, high energy electron beams can induce point defects into the semiconductor structure and suppress the luminescence properties. Moreover, more aggressive specimen preparation methods such as focused ion beam (FIB) lamella preparation, should be used with extreme caution.

A newly developed technique in $\mathrm{CL}$ is spatially-resolved time-correlated (SRTC)-CL, which is capable of generation, detection and characterization of single-photon states in STEM. It takes advantage of fast electrons of the highly coherent and focused (below $1 \mathrm{~nm}$ ) electron probe to excite defect centers, e.g. neutral vacancy centers in diamond, to emit photons and send them to an intensity interferometer, which can measure the second order correlation function, $g^{(2)}(\tau)$. This function provides information about the intensity correlations of a given light field at different time delays, $\tau$. Fig. 3 schematically shows a typical STEM-CL setup and the intensity interferometer. Combination of such high spatial resolution in currently available STEM devices with the capability of detection and characterization of single-photon states and measurement of $\mathrm{g}^{(2)}(\tau)$ function, allows us to address individual quantum bits in compact systems. This can eventually lead to further development of scalable quantum computers $\left[{ }^{64}, 65\right]$.
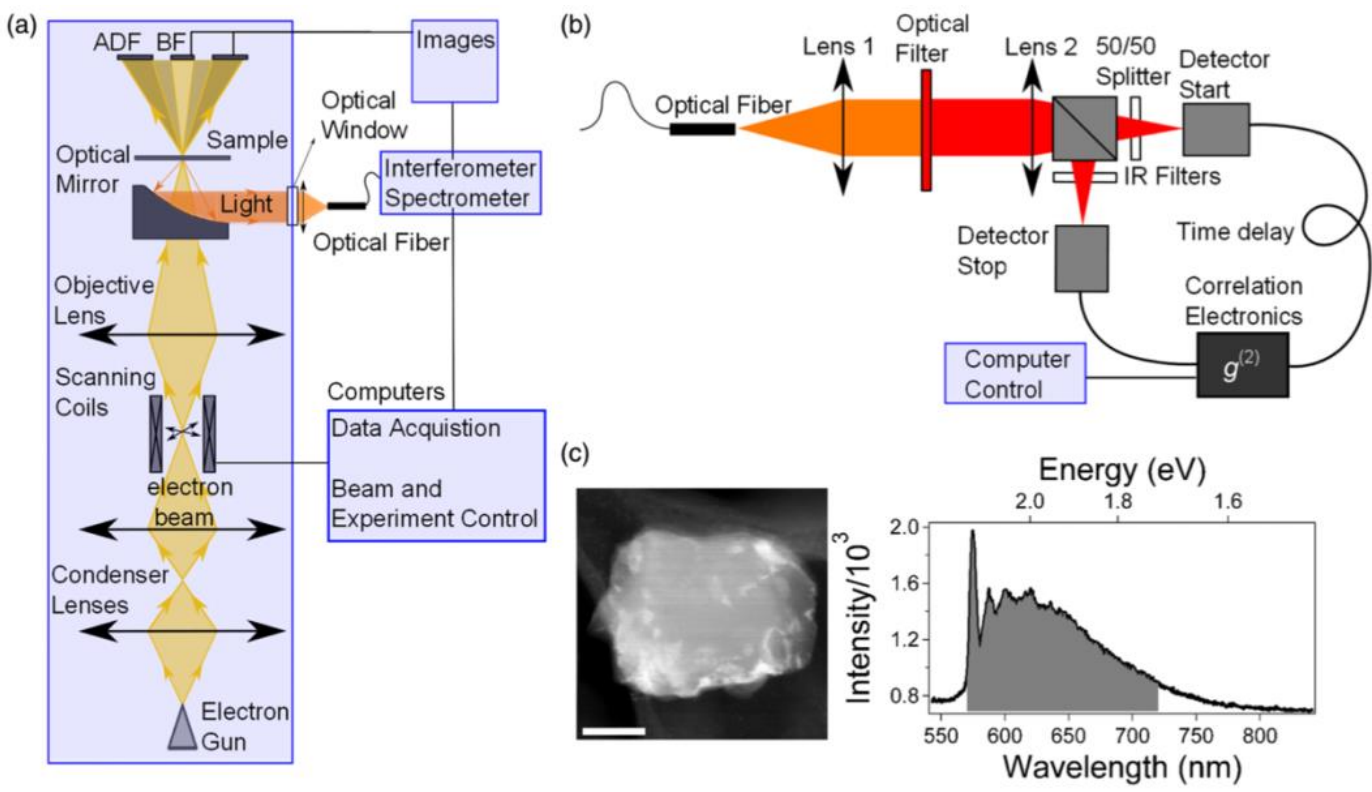

Fig. 3. (a) scheme of STEM-CL setup with intensity interferometer, (b) components of the intensity interferometer, (c) a diamond nanoparticle with the corresponding CL spectrum. Reprinted from [64] with the permission of APS 


\section{STEM applications on 1D nanostructured semiconductors}

In the past decades, the techniques mentioned in the previous section have had dramatic impacts on advancement of materials science, especially semiconductors. In this section we aim to introduce some of the recent applications of these techniques, specifically for $1 \mathrm{D}$ nanostructured semiconductors. Several studies can already be found in the literature. Also, there are promising techniques currently used for other materials (such as oxides) and have a great potential to be employed on semiconductor material systems in order to obtain crucial information and understand their behavior in devices. We start with the main structural issues of semiconductor nanowires, i.e. polarity, growth mechanisms, atomic ordering, structural defects, formation of quantum structures, as well as heterointerfaces, and the lattice strain. Then we move on to the physical properties, electronic bandgap, luminescence, and internal electric field, and explain how they can be measured by means of advanced STEM techniques.

\subsection{Structural properties}

The first application a TEM can have, is resolving the crystal structure. This has been performed traditionally via diffraction techniques and high-resolution TEM (HRTEM) imaging. The polytypes, from wurtzite (WZ) to zinc-blende (ZB), can be identified by HRTEM imaging, having the structure in a suitable zone axis (mainly $[110]_{Z B}-[1-100]_{W z}$ ). STEM imaging, however, offer much more. In the following we will focus on some of the structural aspects that can mainly be studied by atomic-resolution STEM imaging.

\subsubsection{Polarity}

In II-VI and III-V materials, polarity, i.e. the internal electric field caused by the asymmetry in the charge distribution of a cation-anion bond, plays an important role in the physical properties of the compound [ $\left.{ }^{66}\right]$. The question of formation of a 1D semiconductor with a certain polarity, A- or B-polarity, has not yet been answered. Several factors are identified as influential, e.g. the materials themselves, growth environment (chemical potentials, surface energies, precursor flows, temperatures), and the substrates on which the material is grown. However, this discussion is still controversial and needs thorough and systematic investigation $\left[16,{ }^{67}\right]$. Here we bring some examples of the current studies of the polarity of $1 D$ nanostructures.

There are several methods available for determining the polarity, most of which suffer from complication of the method and/or lack of spatial resolution. In contrast, aberration-corrected STEM imaging methods, in particular HAADF and ABF, allow for direct visualization of the socalled dumbbell units, a cation-anion pair. The direction of these dumbbell units (position of the cation and the anion) determines the polarity [15].

In the atomic-resolution HAADF-STEM images of semiconductors from $<110>$ zone axis, as shown in Fig. 4 the dumbbell units can clearly be seen. Since the intensity of the atomic columns depend on their atomic number (the thickness variation in adjacent atomic columns is either zero or negligible, hence it does not contribute), we can extract information about the position of each element. In this example, a GaAs nanowire is shown where As, the slightly brighter element, is on top. Therefore, it can be deduced that the nanowires are B-polar [15]. Polarity assignment in GaAs in particular is difficult (yet possible), since the atomic numbers of $\mathrm{Ga}$ and $\mathrm{As}$ are quite close and hence the intensity difference is small.

The polarity of the 1D nanostructures is often inherited from the substrates on which they grow, especially when they grow vertically. However, II-VI and III-V materials usually have a preferred polarity that is more energetically favorable. Therefore, the growth with the opposite polarity either does not occur or demands extreme conditions. Most of the III-V compounds tend to grow with B-polarity. Atomic-resolution HAADF-/ABF-STEM analysis on B- 
polarity of GaN [ $\left.{ }^{68}\right]$, GaAs $[15,17]$, InAs $\left[{ }^{69},{ }^{70},{ }^{71}\right], \operatorname{lnSb}[69,70]$ and GaSb $\left[{ }^{72}\right]$ have already been reported. In addition, exceptional situations under which nanowires grow with A-polarity, have also been observed $[16,17,67]$. This can be clarified by studying the growth mechanisms, which will be covered in Section 3.1.2.

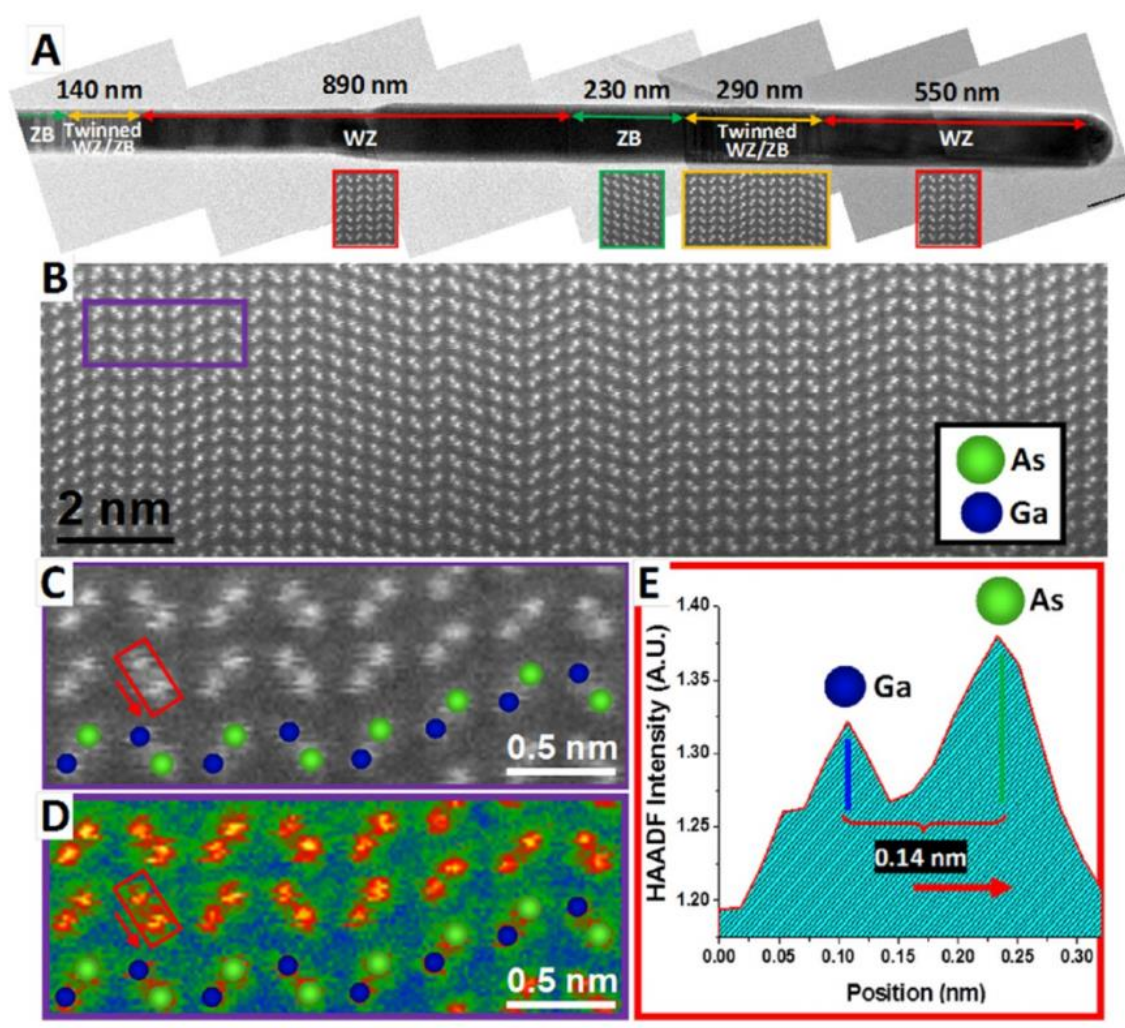

Fig 4. B-polarity in GaAs nanowires, (a) low-magnification TEM image showing the overview of the nanowire, (b) atomic-resolution HAADF-STEM image showing the dumbbell units, $(c, d)$ magninfied HAADF-STEM image indicating the position of $\mathrm{Ga}$ and $A s$ atoms, (e) intensity profile on the indicated dumbbell unit. Reprinted from [15] with the permission of ACS

As mentioned in the previous section, for the compounds such as $\mathrm{ZnO}$ and $\mathrm{GaN}$ which contain light elements, HAADF-STEM does not provide sufficient contrast. The imaging instead can be performed by ABF-STEM. For GaN nanowires N-polarity is predominant. This can be understood when the 1D nanostructures are grown on a non-polar substrate such as $\mathrm{Si}$ or diamond. In Fig. 5 ABF-STEM images of an N-polar GaN nanowire grown on a diamond substrate is revealed [68]. Several other works on GaN and GaN/AIN nanowires also mostly reveal the B-polarity ( $\mathrm{N}$ on top) $\left[15,{ }^{73}\right]$. A $10 \%$ yield of Ga-polar GaN nanowires has also been reported $\left[{ }^{74}\right]$. On the other hand, new methods have been published through which Ga-polar nanowires have also been grown in order to achieve different optoelectronic properties from them. Among all, we mention selective area growth on masked Ga-polar GaN substrates $\left[{ }^{75},{ }^{76}\right]$ and substrate pre-growth treatment $\left[{ }^{77}\right]$. The optoelectronic properties of the former will be discussed in the Section 3.2.2.

The Ga-polar GaN nanowires usually exhibit a pyramidal morphology [ $\left.{ }^{78}\right]$. Apparently, the nanowire growth terminates by formation of (1-102) planes. After the formation of these planes the growth either stops or becomes considerably slow. However, inversion domain boundaries (IDBs) can occur in order for the nanowire to continue growing $\left[{ }^{79}\right]$. Formation of 
an IDB is an energetically costly process. Yet, once it occurs, the nanowire can continue growing with the opposite polarity which conceivably is more energetically favorable.
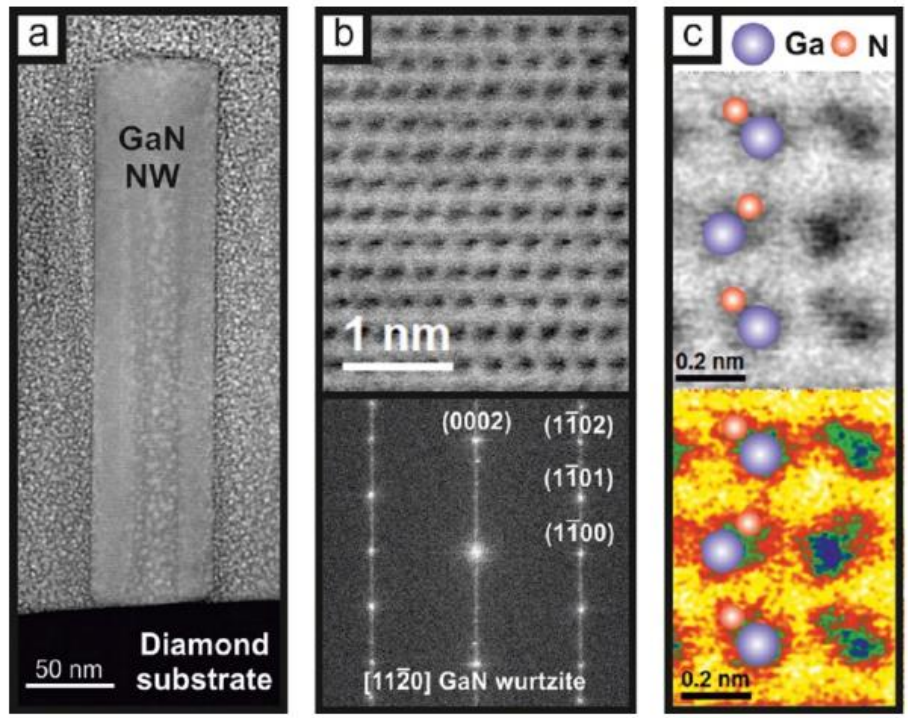

Fig 5. ABF-STEM imaging of N-polar GaN nanowire grown on diamond substrate (a) low-magnification BF-STEM image, (b) atomic-resolution ABF-STEM image with the corresponding power spectrum, (c) more magnified ABF-STEM image showing the Ga-N dumbbell units and the location of each element. Reprinted from [68] with the permission of ACS

$\mathrm{ZnO}$ among II-VI materials, on the other hand, predominantly grows with A-polarity (Zn on top). In the case of van der Waals epitaxy growth on mica substrates with rather weak interaction of the substrate in the growth process, the nanowires are observed to grow with $\mathrm{Zn}$-polarity $\left[15,{ }^{80}\right]$. Even in the case of axial heterostructures where $\mathrm{ZnO}$ is grown on N-polar GaN nanowires, as shown in Fig. 6, ZnO still grows with A-polarity, conceivably causing an IDB at the axial heterointerface. Since the $\mathrm{ZnO}$ nanowire also grows downwards and covers a segment of $\mathrm{GaN}$, the axial heterointerface cannot be clearly identified [66]. Interestingly, occurrence of IDBs in II-VI materials has been frequently observed. It will be elaborated in Section 3.1.4.
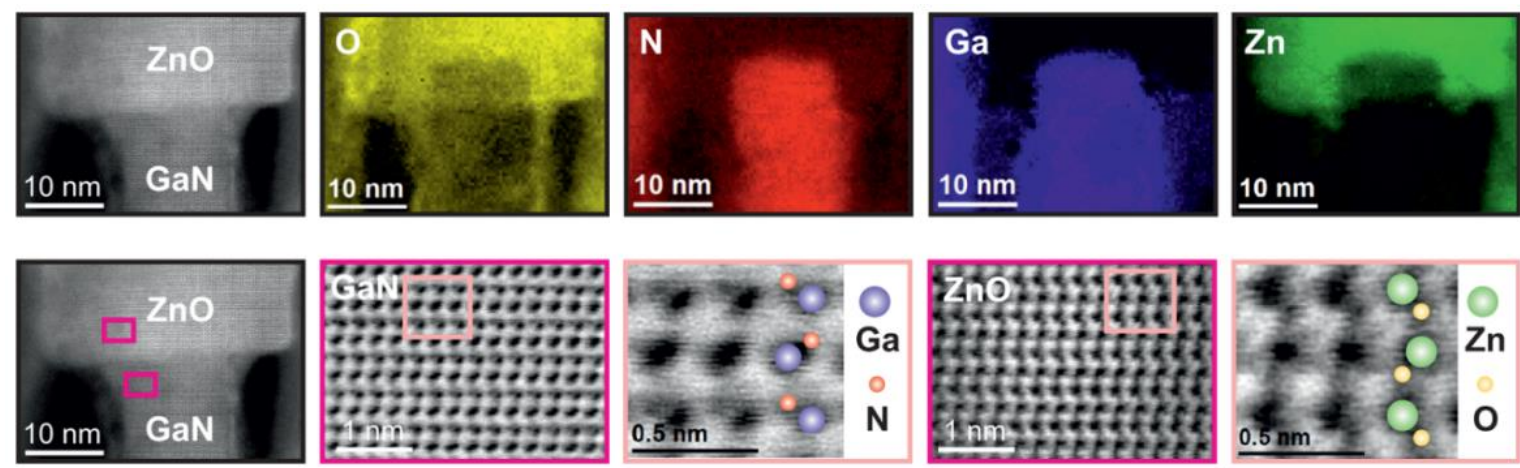

Fig 6. Atomic-resolution ABF-STEM imaging of GaN-ZnO heterostructure nanowire, revealing the B-polarity in $\mathrm{GaN}$ and A-polarity in $\mathrm{ZnO}$, associated with EELS chemical maps. Reprinted from [66] with the permission of ACS

Change of polarity can also occur without any IDB. This usually includes change in growth direction. For instance, the growth of A-polar GaAs nanowires is observed after kinking [17]. 
In the following section we aim to explain the growth mechanism, revealed by STEM imaging, leading to different polarities and morphologies.

\subsubsection{Growth mechanisms}

In order to describe the growth mechanisms clearly, we first need to understand the crystal structure and its relationship with the morphology and the stable facets. In these tetrahedrally-coordinated semiconductors in their zinc-blende polytype (with cubic symmetry), tetrahedron is a common object. It consists of four $\{111\}$ facets of the same polarity with an angle of $109.47^{\circ}$ between the surface normals. Symmetric truncation of a tetrahedron gives rise to formation of octahedron, another common morphology with eight \{111\} facets, half of them A-polar and the other half B-polar. Emergence of tetrahedra/octahedra in solutions or on substrates facilitates the formation of polypods (a socalled polytypic branching phenomenon $\left[{ }^{81},{ }^{82}, 83\right]$ ), or in the case of nanowire growth on substrate, it can give rise to formation of nanowires non-perpendicular to the substrate surface $\left[{ }^{84},{ }^{85}\right]$. This can also lead to formation of nanowires with opposite polarities. Here we aim to explain the growth mechanisms for these phenomena through a few examples.

A simple example is the case of Sn-seeded GaSb nanowires on A-polar GaSb substrates by metal-organic vapor-phase epitaxy (MOVPE). Under certain growth conditions, GaSb nanowires with both A- and B-polarity are present (Fig 4 and 5 of [ $\left.{ }^{86}\right]$ ). By cross-sectional analysis of the sample prepared by focused ion beam (FIB), and correlation of it with SEM images, we understand that prior to the growth of the nanowire, a truncated tetrahedron forms on the substrate below the seed particle as shown in Fig. 7. As can be seen in the 3D atomic models in Fig. 7(a-d), the tetrahedron has three free $\{111\} B$ facets (the fourth is connected to the substrate) all of which are ready for a B-polar nanowire to grow, if the seed particle wets them (Fig. 7(e and g)). These inclined nanowires grow in three possible direction $\left(120^{\circ}\right.$ from each other from the top view), all with an angle of $19.47^{\circ}$ from the substrate. On the other hand, the truncation of the top tip of this tetrahedron avails a \{111\}A facet. If this facet does not shrink in size and the Sn seed particle stays on it, an A-polar nanowire grows vertically. This growth mechanism explains the presence of both A-polar and B-polar GaSb nanowires on the same substrate [86]. 

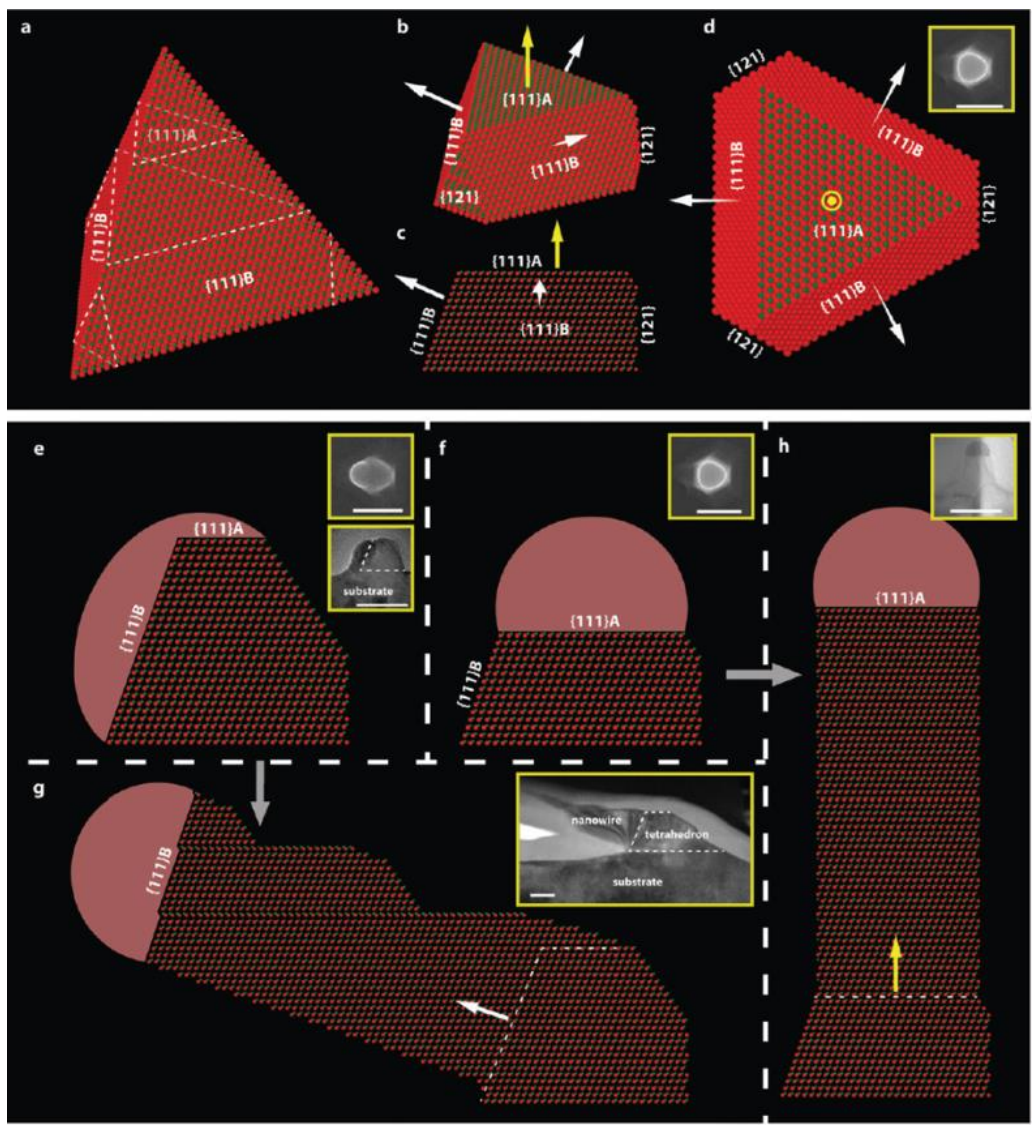

Fig 7. Growth mechanism of GaSb nanowires with both A- and B-polarity, (a) 3D atomic model of a tetrahedron with $\{111\} B$ facets, $(b-d)$ truncated tetrahedron and availability of the $\{111\}$ A top facet; $\{111\}$ A facets shrinks and droplet wets $\{111\} B$ facet, and causes formation of $(g)$ inclined B-polar nanowire; ( $f$ ) droplet stays on $\{111\} A$ facet and forms (h) an A-polar vertical nanowire. Reprinted from [86] with the permission of RSC

Formation of single or multiple octahedra on the substrate increases the possibilities of having inclined nanowires with different angles. This has been reported by Uccelli et al [85]. The GaAs nanowires are grown via molecular beam epitaxy (MBE), self-catalyzed on $\mathrm{Si}<111>$ substrates. In this case, GaAs nanowires grow only with B-polarity, and the angle between the nanowire and the substrate depends on the octahedron $\{111\} B$ facet on which the growth occurs. The growth possibilities on both A-polar and B-polar first and second order octahedra are shown in Fig. 8. As can be seen, with the second order octahedra, angles of $19.47^{\circ}, 33.75^{\circ}$, $51.06^{\circ}$, and $90^{\circ}$ (vertical) are available [85]. 

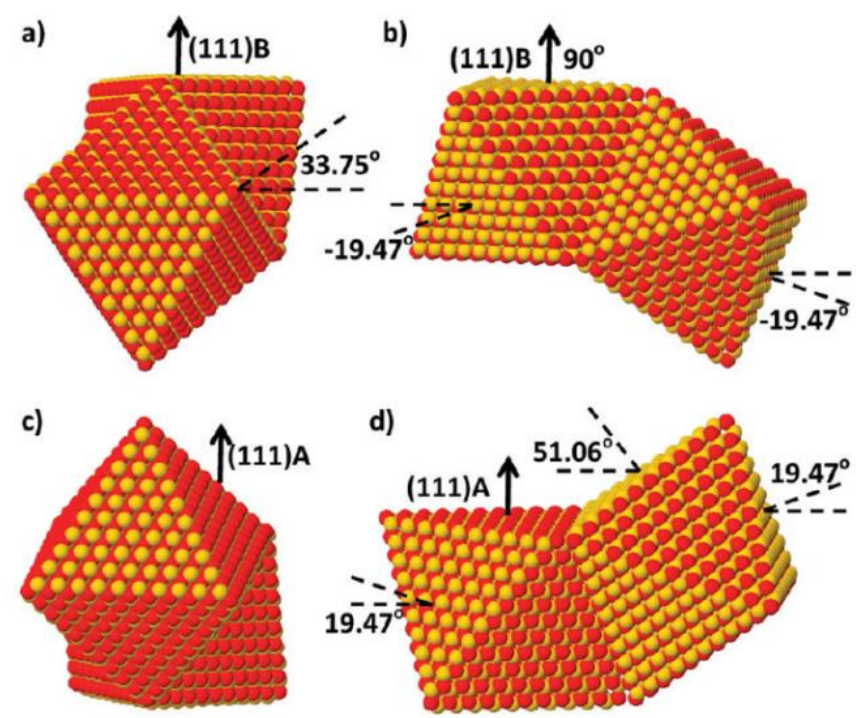

Fig 8. Formation of multiple octahedra and availability of $\{111\} \mathrm{B}$ facets at $19.47^{\circ}, 33.75^{\circ}, 51.01^{\circ}$, and $90^{\circ}$. Reprinted from [85] with the permission of ASC

In that study formation of A-polar GaAs nanowires had been considered impossible. However, more recently the yield of MBE-grown A-polar GaAs nanowires has been increased up to $\sim 50 \%$ by using on GaAs $\{100\}$ substrates and employing highly Ga-rich conditions which influences the $\{111\} A$ and $\{111\} B$ surface energies. By tweaking the surface energies, one can increase the wettability of the A-polar surfaces of the tetrahedron/octahedron which gives rise to A-polar nanowire growth. It is worth noting that in this case, the contact angle of the droplet with the nanowire, which is known as an influencing parameter in growth with different polarities, was similar for A- and B-polar nanowires. As expected, neither A- or Bpolar nanowires grow perpendicularly on the GaAs $\{100\}$ substrates. They both grow with an angle of $54.5^{\circ}$, and from the top view, the nanowires with opposite polarity seem perpendicular with respect to one other [67].

Another important phenomenon is kinking of the nanowires, where the growth direction changes. Intentional kinking has also been reported where the aim for this process is to make the nanowires meet each other and create epitaxial crosses that can be used to study fundamental physics such as Majorana fermions $\left[{ }^{87},{ }^{88}\right]$. Kinking is usually associated with formation of one twin boundary. It can either change the growth direction to a completely different crystallographic orientation, e.g. from $<100>$ to $<111>B$ and back to $<100>$ in InP nanowires $\left[{ }^{89}\right]$, or more commonly observed, it changes the polarity, e.g. from $<111>A$ direction to $<111>B$ in GaAs nanowires. In Fig. 9 a kinked GaAs nanowire is analyzed by means of atomic-resolution HAADF-STEM imaging. It is shown that after occurrence of a single inclined twin boundary the nanowire kinks from $<111>A$ to $<111>B$ direction. The wetting angle of the droplet is considered as the main parameter. It is also observed that the structural properties of the A- and B-polar segments of the nanowires are different. As can be seen in Fig. 9(b,c), twin boundaries occur more frequently in the B-polar segment [17]. 

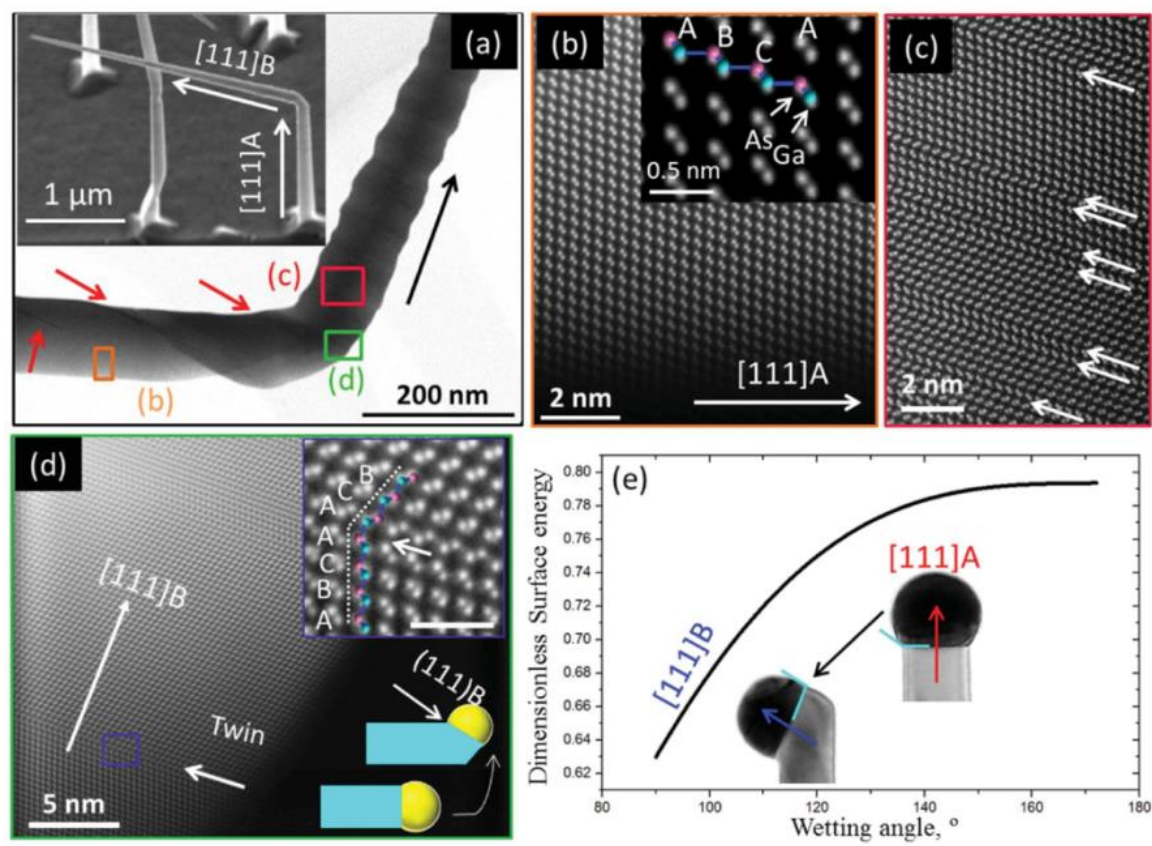

Fig. 9. Kinking of an A-polar GaAs nanowire caused by a twin defect, (a) SEM and TEM images showing the overview of the kinked nanowire; atomicresolution HAADF-STEM image showing (b) the defect-free structure of the Apolar part, and (c) defected structure of the B-polar part after the kink, (d) the twin boundary that causes the kink and change of polarity from $A$ to $B$, (e) diagram of wetting angle vs. surface energy. Reprinted from [17] with the permission of Wiley

As discussed, nanowire growth mechanism, polarity, and formation of structural defects are interrelated. Therefore, it is important to be able to observe the structural defects (as well as polarity) and understand their relationship with the morphology in order to be able to tailor the nanostructures at will. In the following we discuss the realization of structural defects by atomic-resolution HAADF-STEM imaging, and the challenges to disclose the hidden ones.

\subsubsection{Structural defects}

Twin boundary - Twinning is the most common type of stacking faults in tetrahedrallycoordinated semiconductors. Twin boundaries, occurring in $\{111\}$ planes, change the stacking sequence of the planes without causing disruption to the tetrahedral bonding. Such structural defect should be avoided since they often deteriorate the physical properties of the semiconductor $\left[{ }^{90}\right]$. In contrast, in some particular cases such as in thermoelectric devices they can even enhance the properties (thermoelectric figure of merit, zT) by increasing the phonon scattering $\left.{ }^{[91}\right]$.

Identification of twin boundaries is fairly easy, and has been performed by conventional HRTEM and diffraction pattern methods for many decades [ $\left.{ }^{92}\right]$. However, it is still important to take into account that from a specific orientation, i.e. $<112>Z B$ and $<12-30>W Z$ which is a main pole in these structures, the stackings of $\{111\}$ planes cannot be distinguished. This is a common mistake to have a nanowire aligned along the mentioned zone axes, and believe that it is defect-free, although neither the crystal structure (wurtzite or zinc-blende) nor the stacking faults and twin boundaries can be possibly observed from this direction. 
It should be pointed out that the ease of observation of the stacking faults is till their plane normal is perpendicular to the zone axis. The ones in different orientations, in contrast, are not clearly observable, and more importantly, can cause confusion in identification of the crystal structure. It is explained how one twin boundary in a Si nanoparticle with its normal along the zone axis, or a 'hidden' defect (or grain boundary) in zinc-blende Si nanowires can create extra reflections in the diffraction patterns and be misunderstood with wurtzite structure $\left[{ }^{93},{ }^{94}\right]$. Simulated HRTEM image of the Si nanoparticle with (top) and without (bottom) a twin boundary in the middle, shown in Fig. 10 obtained from ref. [93], reveals how misleading the reflections can be (top raw except the first at right).

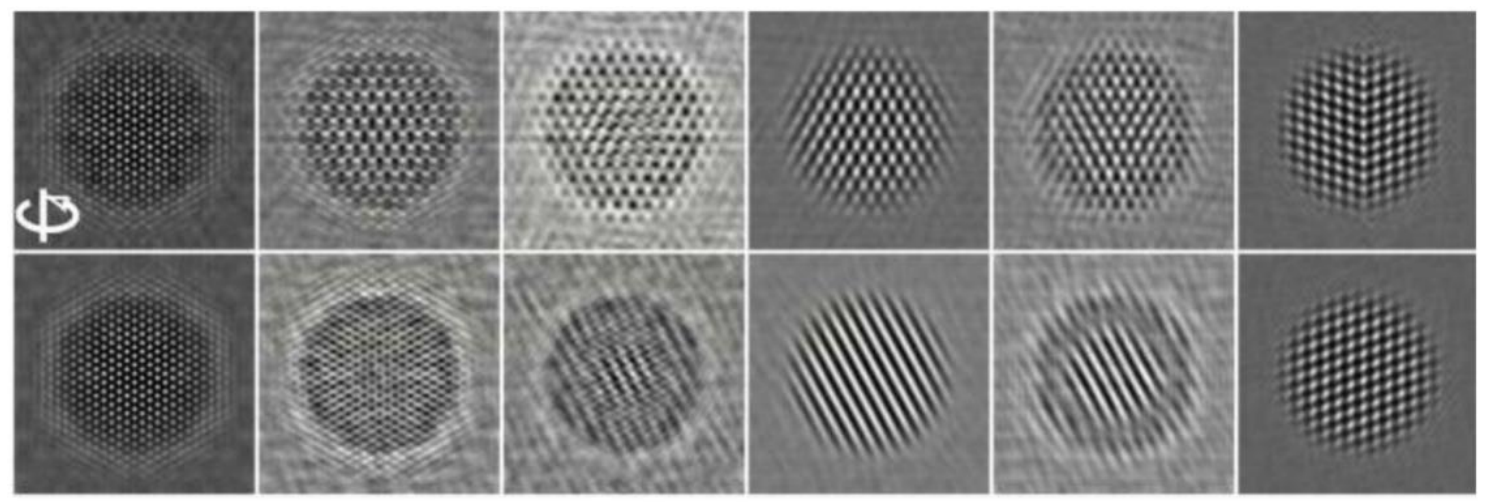

Fig. 10. Simulated HRTEM image of the Si nanoparticle with (top) and without (bottom) a twin boundary in the middle. The twin boundary can be clearly observed from <110> direction (top-right-most), but rotating from 0 to $90^{\circ}$ $(<111>$ direction), the reflections are misleading. Reprinted from [93] with the permission of Wiley

In B-polar III-V nanowires, formation of twin boundaries in growth direction is common, and their population (which can lead to change of crystal structure from zinc-blende to wurtzite or vice versa) can be controlled by tweaking the growth parameters such as the flows and $\mathrm{V} / \mathrm{III}$ ratios $\left[24,{ }^{95},{ }^{96}\right]$. Twin boundaries, however, can also form in any $<111>$ direction other than the growth direction. This usually means that they appear along the opposite polarity. Formation of one single inclined twin boundary often leads to kinking and change of polarity and growth direction, as shown in Fig. 9 and discussed in Section 3.1.2 for the case of Au-seeded MOVPE-grown GaAs and GaAsSb nanowires. It is interesting to note that the density of twin defects in the A-polar part of the nanowire is quite low, and only few inclined defects can be observed which in some cases give rise to kinking. The B-polar part of the nanowire, in contrast, is much more defected [17]. The same phenomenon has also been observed more recently in self-seeded MBE-grown GaAs nanowires. In this case, the A-polar nanowires appear to be defect-free while the B-polar ones contain several twin boundaries along the growth plane [67].

In Sn-seeded GaSb nanowires also no twin defects have been observed in A-polar nanowires, while their B-polar counterparts exhibit inclined twin boundaries. In the latter case, formation of the inclined twin boundaries always occurs in pairs, along \{111\}A planes. This affects the morphology of the nanowires by causing some steps in the lateral facets. These inclined twin boundaries are not always perpendicular to the view direction. They can also intersect in the nanowire structure when they occur in different $\{111\} A$ planes. In this study has been shown that by aberration-corrected HAADF-STEM methodology one can identify the presence of the inclined twin pairs that are not in the zone axis. As shown in Fig. 11(b) presence of the inclined twin pair, with $60^{\circ}$ angle to the zone axis, causes an extra reflection in between two dumbbell units (yellow inset) which does not exist in a defect-free 
zinc-blende structure (green inset). The simulated HAADF-STEM image shown in Fig. 11(c), taken from the 3D atomic model in Fig. 11(d), confirms the presence of such hidden twin boundaries [86].
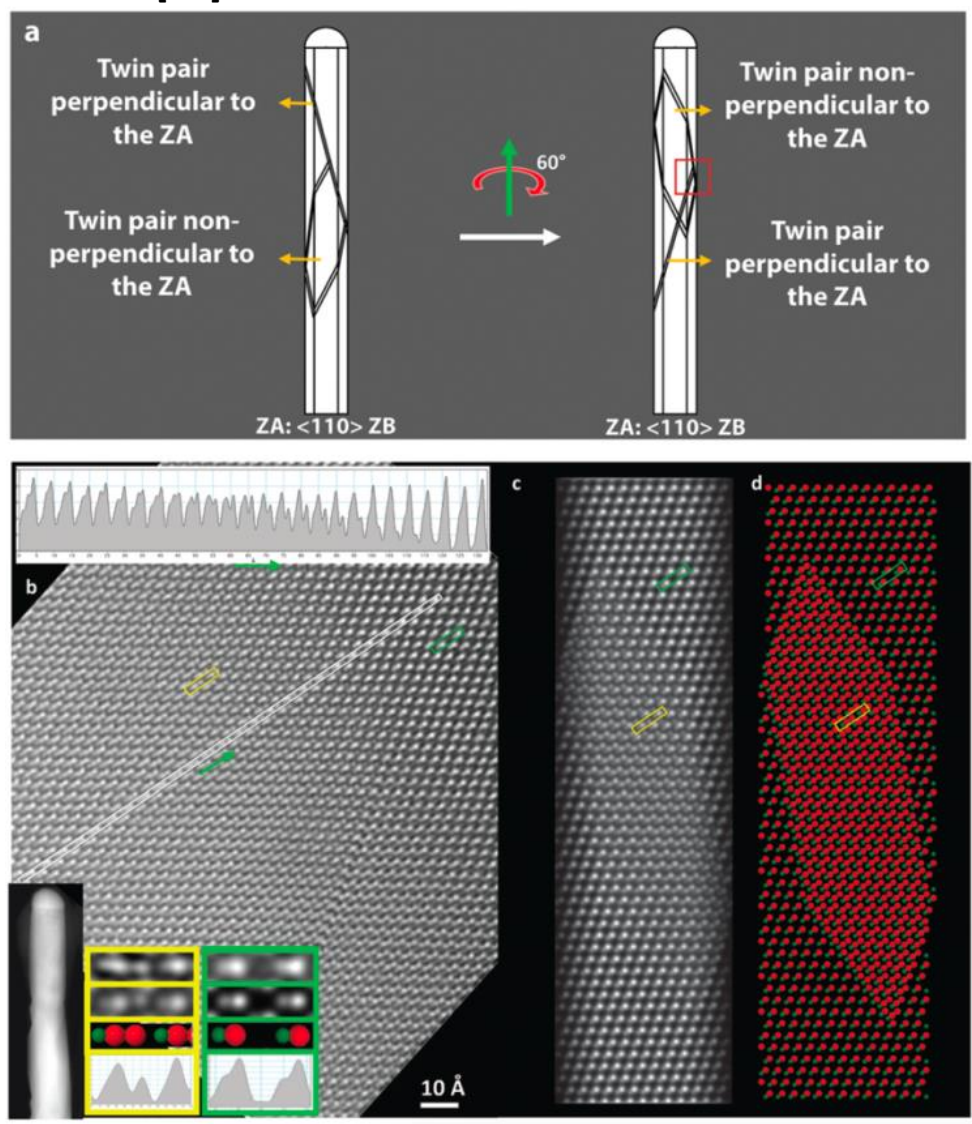

Fig. 11. Identification of hidden twin boundaries in B-polar GaSb nanowires. (a) schematic illustration of twin pairs with a $60^{\circ}$ angle with respect to $\langle 110\rangle$ zone axis. (b) experimental and (c) simulated atomic-resolution HAADF-STEM images on such twin boundaries, shown in (d) 3D atomic model; the yellow insets in (b) show the presence of an extra reflection caused by those hidden twin pairs, which is absent in the green inset taken from a defect-free zinc-blende structure. Reprinted from [86] with the permission of RSC

We just mentioned that the single inclined twin boundaries can cause kinking and hence change in polarity. This change, however does not include any inversion domain boundaries (IDBs). In the following we discuss some other cases in which IDBs actually emerge.

Inversion domain boundary - Although IDBs in general do not occur often in 1D nanostructure semiconductors (since their formation requires high energies), their emergence in $\mathrm{ZnO}$ has been reported several times, especially when doped with trivalent metals such as In, Ga, and Fe $\left[{ }^{97}, 98,99,100,101\right]$. The dopants in $\mathrm{ZnO}$ structure create a unique superstructure by lining up along (0001) and (2-1-15) planes of $\mathrm{ZnO}$ and creating a homogeneous $\mathrm{M}_{2} \mathrm{O}_{3}$ ( $\mathrm{M}$ : trivalent metals) layer between two layers of $\mathrm{ZnO}$ and separating domains with opposing polarities. Two types of IDBs are formed in ZnO: basal IDBs, b-IDBs, on (0001) planes, and pyramidal IDBs, p-IDBs on (2-1-15) planes. In the b-IDBs, the trivalent atoms are in octahedral coordination with the surrounding $O$ atoms. These $O$ atoms are still tetrahedrally coordinated, but here by three trivalent atoms and one $\mathrm{Zn}$ atom [97]. The atomic models in Fig. 12 (right) reveal the structure of $b$ - and p-IDBs and the way they cause change in polarity. The yellow spheres are $\mathrm{O}$, grey ones are $\mathrm{Zn}$, and the blue ones are the trivalent 
metals (In in this case). The image at left is a TEM micrograph of a $\mathrm{ZnO}$ nanowire doped with In, in which the b-IDBs and p-IDBs are penetrating from the side facets into the nanowire [98].
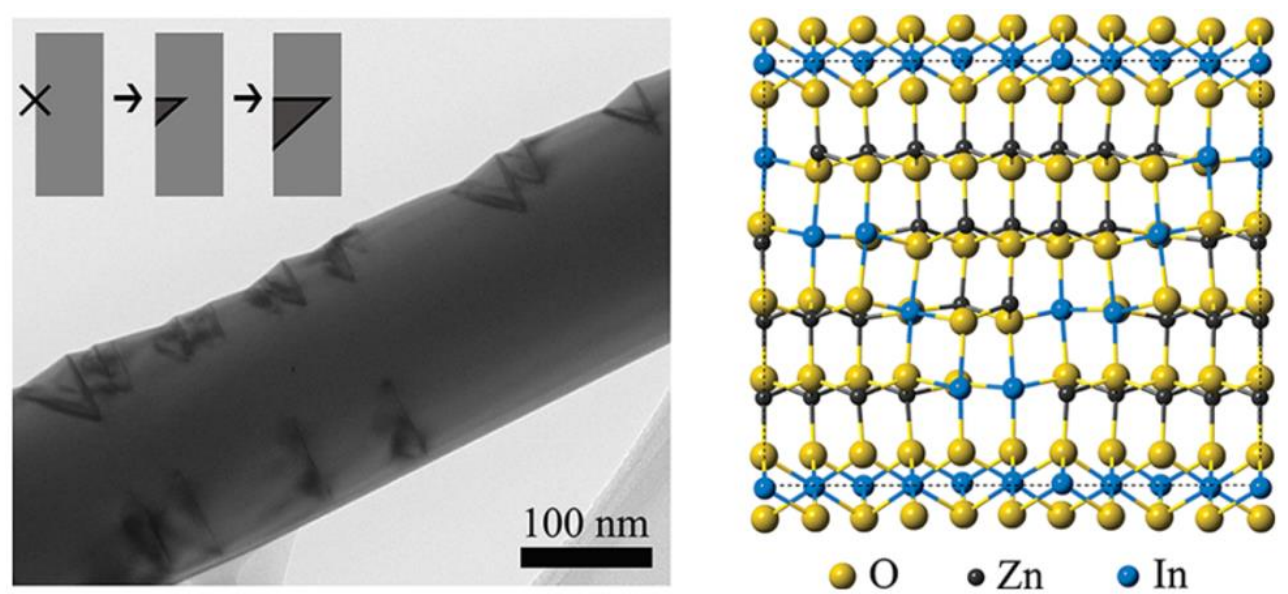

Fig. 12. (left) TEM image of an In-doped ZnO nanowire containing $p$ - and b-IDBs, (right) 3D model of the atomic arrangement of the IDBs. Reprinted from [98] with the permission of ACS

Fig. 13 shows an atomic-resolution ABF-STEM image of a b-IDB in an In-doped ZnO nanowire. The position of $\mathrm{In}, \mathrm{Zn}$ and $\mathrm{O}$ atoms are shown by red, green and blue circles. In atoms (the heaviest) appear considerably darker since the contrasts in ABF-STEM images are reversed. Note that imaging of $\mathrm{O}$ atomic columns would not be possible by HAADF-STEM imaging, since the Z-contrast of such light element is not visible in the vicinity of $\mathrm{Zn}$ and $\mathrm{In}$. As can be seen, the segment of the nanowire below the $b$-IDB is A-polar ( $Z n$ on top in the dumbbell units) while above the b-IDB the polarity is inversed [97]. It is shown that presence of IDBs in the $\mathrm{ZnO}$ structure has a dramatic impact on its physical properties, an example of which is the improvement of the thermoelectric figure of merit in $\ln _{2-x} \mathrm{Ga}_{x} \mathrm{O}_{3}(\mathrm{ZnO})_{n}$ nanowires by 2.5 order of magnitude in comparison to undoped $\mathrm{ZnO}$ nanowires [99].
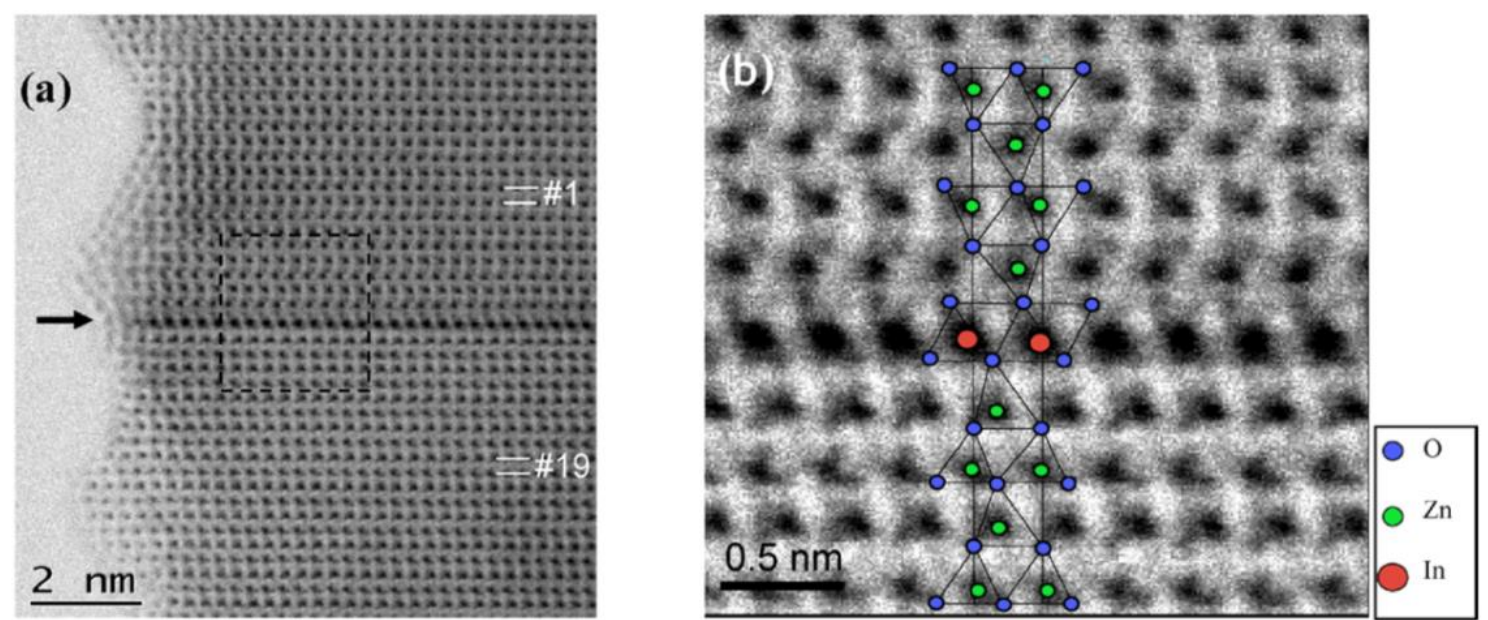

Fig. 13. (a) Atomic-resolution ABF-STEM image of a b-IDB in an In-doped ZnO nanowire, (b) further magnified ABF-STEM image showing the atomic arrangement at the b-IDB and position of each element. Reprinted from [97] with the permission of Elsevier 
Other defects, grain boundary - Apart from twin boundaries, more complicated defects can occur in the semiconductor nanostructures, especially when twin boundaries or crystals with different orientation or symmetry meet $\left[88,{ }^{102}\right]$, or the twin boundaries do not cross the whole width of the nanowires or become stepped. Here we aim to describe such defects. Some of these defects are thoroughly studied by Sanchez et al in GaAs and GaAsP nanowires $\left[{ }^{103},{ }^{104}\right]$. The atomic-resolution HAADF-STEM images in Fig. 14 indicate a stepped twin boundary which creates a typical $\Sigma 3$ (11-2) interface (para-twin) consisting in a 7-membered (bottom) and a 5-membered (top) rings. It can clearly be seen that the twin boundary on the left side of the image is six atomic bilayers above the right side, giving rise to formation of two sets of rings. These rings contain over-bonded (Ga-As-Ga) and under-bonded (As-As) atoms, which can act as a nonradiative recombination center having a considerable impact on the electronic properties of the nanowires, therefore, need to be passivated. [103]. Such defects have also been reported by other researchers in MBE-grown GaAs nanowires [67].
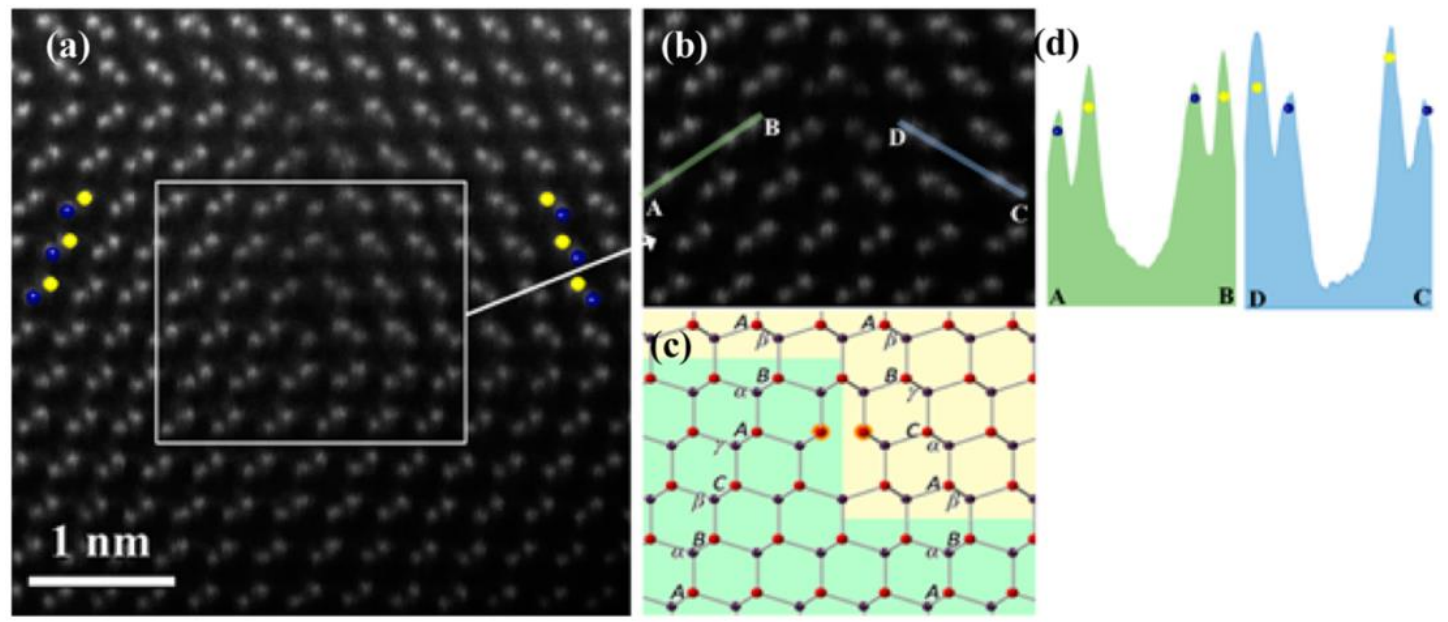

Fig. 14. (a,b) Atomic-resolution HAADF-STEM image of a $\Sigma 3$ (11-2) interface (para-twin) in a GaAsP nanowire, (c) atomic model showing the position of each element, blue for group III and red for group V elements, (d) intensity profiles along two dumbbell units, showing the mirroring along the para-twin. Reprinted from [103] with the permission of ACS

In addition, it is observed that sometimes in the $\Sigma 3$ (11-2) interfaces, the middle plane dumbbells, and the top/low atoms of the dumbbells of the lower/upper (111) planes become blurry from the defect to one side. This is in fact the case of a twin that is terminated by a $\Sigma 3$ (11-2) facet which is not along the zone axis and has an angle of $60^{\circ}$ with it [103].

Other types of defects are also observed in GaAsP nanowires, and have been clearly identified by a Burgers circuit protocol, and their stability is discussed based on their dislocation characteristic deduced from the Burgers vectors. In Fig. 15(a) the same threeatomic-bilayer defect is marked by yellow circles. Since the Burgers vector for it is zero, it has no dislocation character and therefore is stable and has no long-range strain field. In contrast, the Burgers vector of the upper part with two atomic bilayers is not zero. In Fig. 15(b), on the left, the red and blue circles mark two defects that are in fact, respectively, the lower and the upper parts of the same defect (yellow circles in Fig. 15(a)). Analyzing each of them separately, the lower defect (one atomic bilayer, intrinsic stacking fault) and the upper one (two atomic bilayers, extrinsic stacking fault) both have long-range strain fields. These fields, however, cancel each other out if analyzed together. The defect on the right side of Fig. 15(b), and the one in Fig. 15(c) also have the same nature as the ones with the same colors, but doubled in size and their Burgers vectors are zero. The defect in Fig. 15(d) however contains an additional feature resulted from intersection with another defect non-perpendicular to the zone axis. 
Presence of such structural defects in general deteriorates the optical properties of the nanowires and need to be avoided [104].

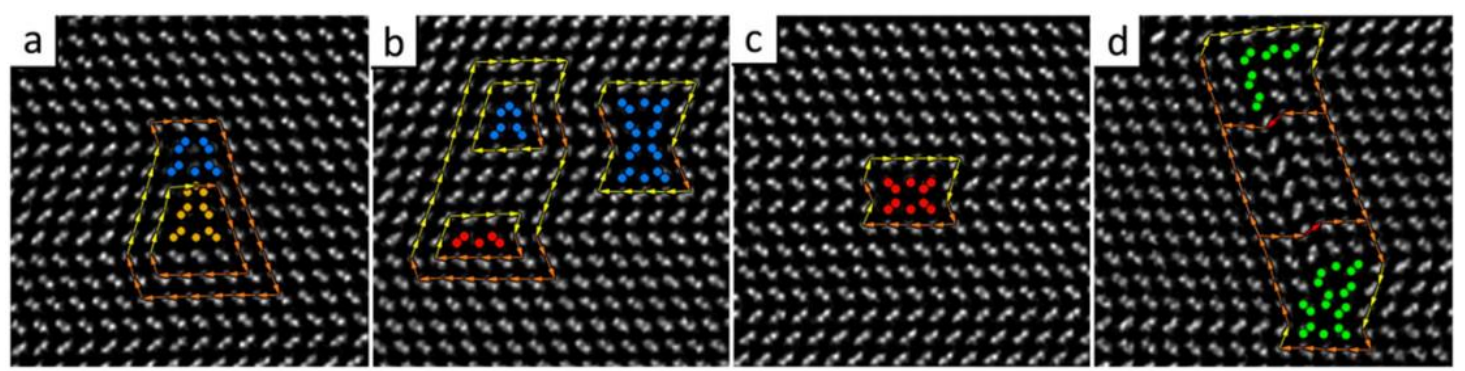

Fig. 15. Atomic-resolution HAADF-STEM image of various types of stable defects in a GaAsP nanowire, analyzed by a Burgers circuit protocol. Reprinted from [104] with the permission of ACS

Point defect, single atoms - Incorporation of Au seed impurities degrades the electronic properties of the semiconductors and is in general to be avoided for applications such as charge transport and optoelectronics [ $\left.{ }^{105}\right]$. Homogeneous coating of Si nanowires with $\mathrm{Au}$ nanoclusters, in contrast, has been proven particularly efficient as bio-sensors with unique capability of detecting breast cancer cell. The homogeneous distribution of Au nanoclusters on these thermal-CVD-grown Si nanowires is shown in the low-magnification HAADF-STEM image in Fig. 16(a). Incorporation of $\mathrm{Au}$ at the atomic level is revealed by analyzing the intensity profiles obtained on the dumbbell units in the aberration-corrected HAADF-STEM image in Fig. 16(b). The additional brightness of the atomic columns in Si dumbbells indicate the presence of Au atoms, since the intensities are supposed to be uniform in the case of pure Si $\left.{ }^{106}\right]$.
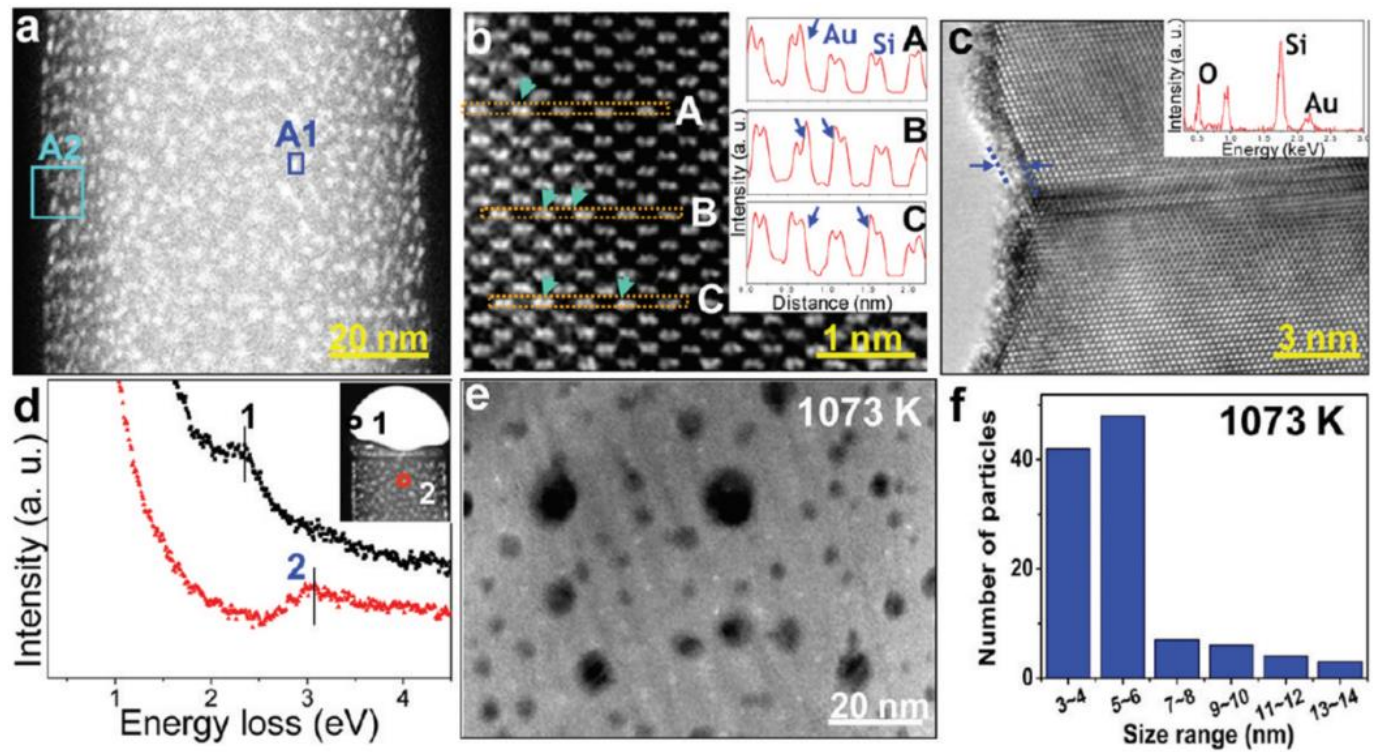

Fig. 16. (a) HAADF-STEM image showing the distribution of Au nanoparticle on the surface of a Si nanowire, (b) atomic-resolution HAADF-STEM incorporation of $\mathrm{Au}$ atoms in the structure of Si matrix, (c) HRTEM image showing Au at the surface and the corresponding EDX spectrum as inset, (d) low-loww EELS signals on the Au alloy droplet and the SI nanowire, (e) BF-TEM image after thermal annealing, (f) Histogram of Au nanoparticle size distribution after annealing. Reprinted from [106] with the permission of ACS 
Aberration-corrected HAADF-STEM imaging is even capable of identifying single atoms in a semiconductor structure. Bar-Sadan et al introduce a methodology through which a single $\mathrm{Au}$ atom can be located in a GaAs matrix with atomic precision. By tilting slightly from the zone axis, the so-called channeling effect and consequently the effect of several material and instrument parameters are reduced. Therefore, one can observe the exact location of the $\mathrm{Au}$ atom, by reducing the background signal of the adjacent $\mathrm{Ga}$ atoms caused by high angle scatterings, and hence enhancing the Au signal. Fig. 17 shows the presence of single Au atoms close to the Au seed particle (Fig. 17(a,b)) and in the middle of the nanowire (Fig. 17(c,d)) [ $\left.{ }^{107}\right]$.
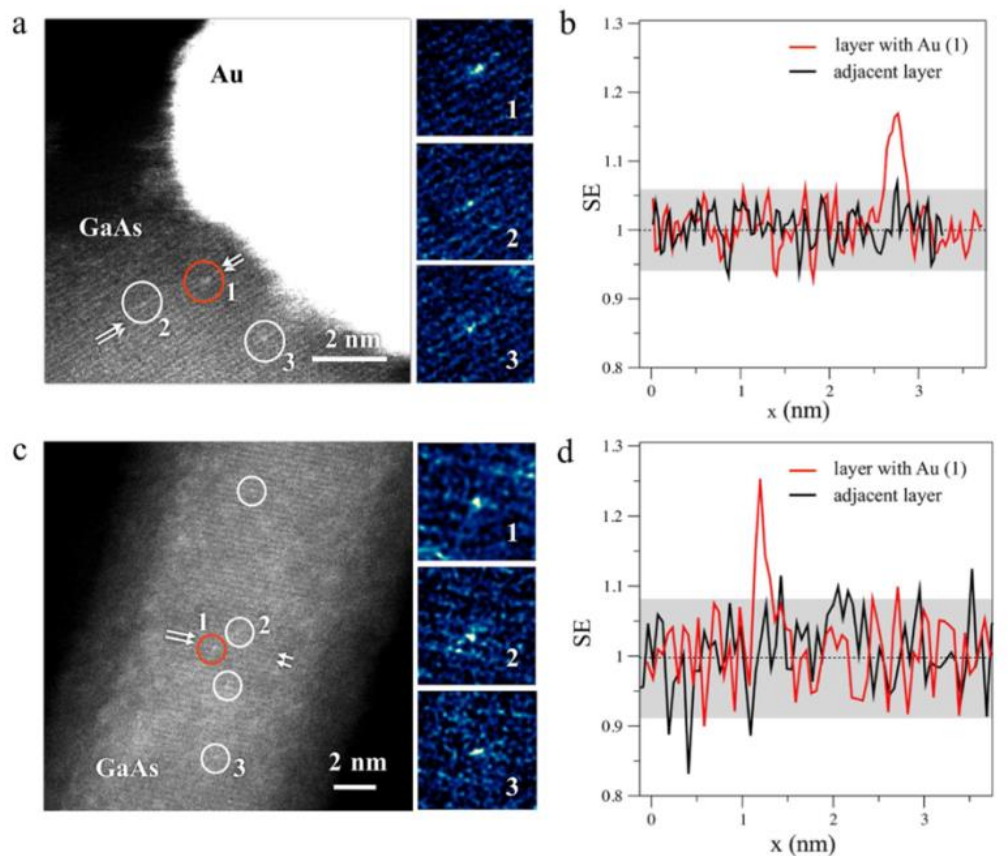

Fig. 17. Single Au impurity atoms in GaAs nanowires; $(a, c)$ atomic-resolution HAADF-STEM images of GaAs nanowires slightly off zone axis to avoid contribution of the channeling effect; $(b, d)$ intensity profiles along the lattice planes that contain the $\mathrm{Au}$ atom and an adjacent plane, indicated by white arrows. Reprinted from [107] with the permission of ACS

Another method for identification of single atoms is core-loss EELS in combination with aberration-corrected STEM imaging. It has been employed recently to observe 1D ionic chains of caesium iodine ( $\mathrm{Csl}$ ) in carbon nanotubes. The difference in intensity of Cs and I can be distinguished in atomic-resolution HAADF-STEM images, although their atomic numbers are quite close $\left(Z_{I}=53, Z_{c s}=55\right)$. Core-loss EELS mapping, in addition, provides chemical signal on each atomic position. Spectrum maps of the $C_{s} M_{4,5}$ edge starting from $750 \mathrm{eV}$ and the I $M_{4,5}$ edge starting from $670 \mathrm{eV}$, reveal the exact position of each single atom of Cs and $\mathrm{I}$, respectively [108].

Spectrum imaging by means of EELS map, as seen here and as mentioned in Section 2, is a powerful technique to study different structural aspects of complex nanostructure materials. In Section 3.1.6 we bring an example of EELS spectrum imaging application on heterostructure III-V nanowires, providing information on atomic arrangement of the axial and radial heterointerfaces with sub-angstrom resolution. Before, we review the phenomenon of atomic ordering, and how it can be revealed by atomic-resolution STEM imaging techniques. 


\subsubsection{Atomic ordering}

Atomic ordering in ternary or quaternary semiconductors has been proven to have a dramatic effect on electronic band structure $\left[{ }^{109}, 110,111\right]$. Several studies have theoretically and experimentally shown the difference of the physical properties between ordered and disordered structures such as InGaAs, GaAsSb, InGaP [ $\left.{ }^{112}\right]$, AllnP [ $\left.{ }^{113}\right]$ and AIGaInP [ $\left.{ }^{114}\right]$

Previously, the use of scanning tunneling microscopy (STM) to identify atomic ordering has been reported [ $\left.{ }^{115}\right]$. Conventional HRTEM imaging associated with diffraction pattern methods also can provide information about the atomic ordering $[116,117,118,119,120]$. However, they might lack accuracy and spatial resolution when it comes to the nanostructures and small differences in atomic number. Atomic-resolution STEM imaging, in contrast, can provide precise, direct and unambiguous evidence on the presence of ordering. It has been used for identifying atomic ordering in perovskite thin film (quantitative STEM imaging) [ ${ }^{121}$ ] and GaAsBi layers [ ${ }^{122}$ ] and $\mathrm{Cu}_{2} \mathrm{CdSnSe}_{4}$ (CCTSe) quaternary chalcogenide polypods [83]. In the following the latter case will be elaborated in detail.

Quaternary CCTSe with diamond-like (zinc-blende) structure consists in four elements, three of which, $\mathrm{Cu}, \mathrm{Cd}$ and $\mathrm{Sn}$, place in the cation position and one, $\mathrm{Se}$, in the anion position. The cations, however, can have different arrangements. They can be randomly distributed leading to a normal zinc-blende structure, or they can be atomically ordered, giving rise to formation of stannite, kesterite or primitive mixed CuAu (PMCA) crystal structures $\left[{ }^{123}\right]$. The unit cells of these structures as shown in Fig. 18 are similar to the one of zinc-blende with this difference that the atomic ordering of the cations doubles the size of it in $c$ direction, and the difference between these three crystal structures is obviously the way the cations are arranged [83].

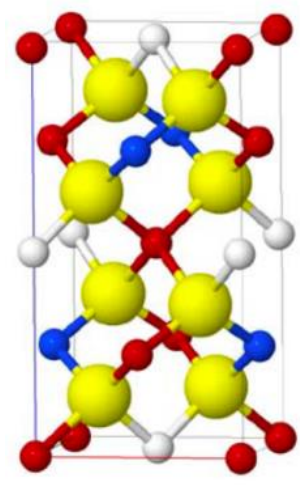

Kesterite

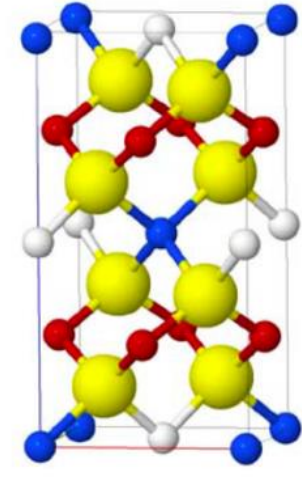

Stannite

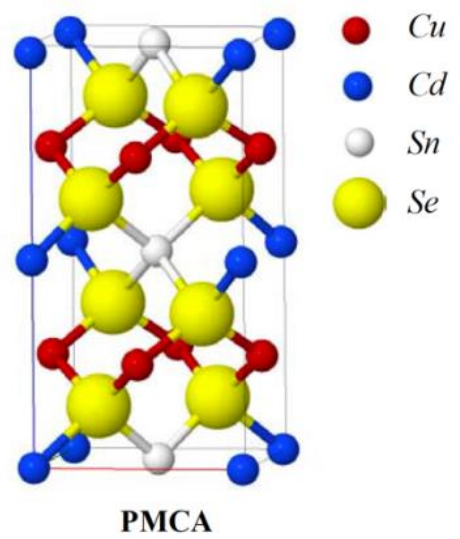

PMCA

Fig 18. Unit cells of kesterite, stannite, and PMCA structures of $\mathrm{Cu}_{2} \mathrm{CdSnSe} 4$ and the atomic ordering of $\mathrm{Cu}, \mathrm{Cd}$ and $\mathrm{Sn}$ in cation sites. Reprinted from [123] with the permission of UB

Fig. 19 shows atomic-resolution HAADF-STEM images of the base of a CCTSe monopod. This base is a pentatetrahedral (a tetrahedron that has four tetrahedra grown on each of its facets) shape a zinc-blende-like structure, and one of its tips has branched out via polytypic branching, i.e. a branch with wurtzite structure. The atomic-resolution HAADF-STEM images reveal that the cations are atomically ordered and the structure is stannite. This is extrapolated from the intensity profile taken along the dumbbell units visualized from [111] (equivalent to $<211>$ ZB) zone axis. The intensities are relative to the atomic number of the cations. Therefore, the brightest points show the position of Sn (white balls), and they become darker for $\mathrm{Cd}$ (blue balls) and then darker $\mathrm{Cu}$ (red balls). For better clarity, the image is magnified (Fig. 19(b)), deconvolved (Fig. 19(c)) and corroborated with linearly-simulated STEM image (Fig. 19(d)) [44] using the 3D atomic models (Fig. 19(e)) [48]. It is worth noting that 
visualization of cation ordering, as firm evidence for formation of stannite structure, is only possible from [111] zone axis. Even it cannot be performed from [241] direction which is another equivalent to $<211>\mathrm{ZB}$, because $\mathrm{Sn}, \mathrm{Cd}$ and $\mathrm{Cu}$ mix up in the cation atomic columns. As can be seen in Fig. 19(g), for the same reason neither [110] or [021] directions (both equivalent to $<011>$ ZB) can be used for this purpose [83].

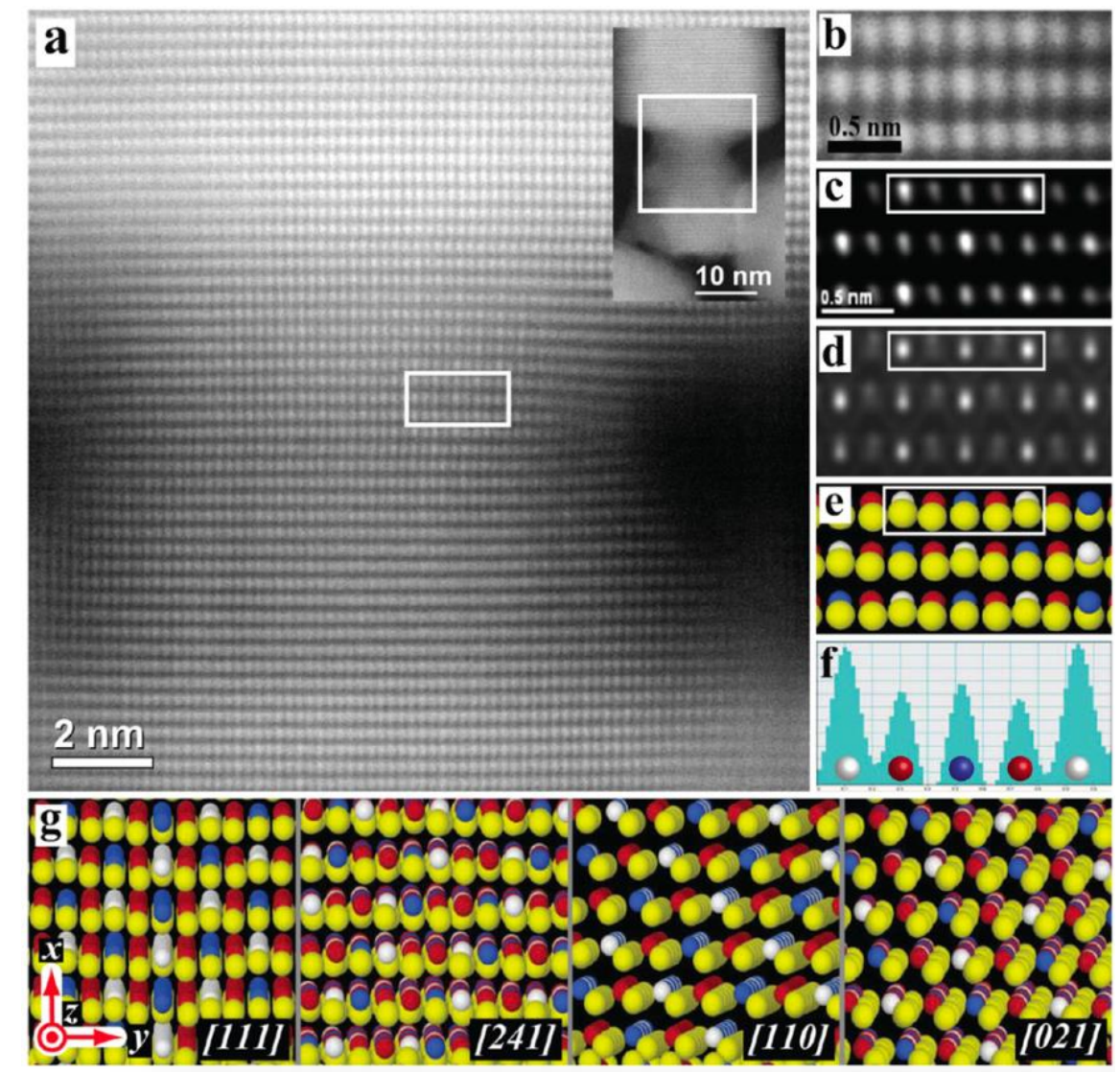

Fig 19. Atomic ordering of the cations in CCTSe polypods; (a) atomic-resolution HAADF-STEM image on the zinc-blende-like base, (b) magnified, (c) deconvolved, (d) simulated HAADF-STEM images, (e) 3D atomic model, (f) intensity profile on the indicated white box; (g) [111] is the only suitable zone axis from which ordering can be observed. Reprinted from [83] with the permission of ACS

Another example of realization of atomic ordering is the case of InGaN quantum dots imbedded in GaN nanowires. Fig. 20(a) shows an ABF-STEM image of an ordered InGaN quantum dot. The indicated part of this image is magnified in Fig. 20(b) from which the intensity profiles are obtained (Fig. 20(c)). As can be seen in these profiles, a clear atomic ordering of Ga-rich and In-rich sequential planes exists in these quantum dots, through which the material compensates for structural strain and lowers the overall energy of the system. As the InGaN quantum dot is accommodated in the GaN matrix under certain amount of compressive strain, it is perceived that such ordering results in better bond relaxation, since different covalent radii of In and Ga atoms can impart slight compressive and tensile strains into their respective In-rich and Ga-rich layers. Extracted EELS signal also confirms the presence of ordering in the quantum dots. The In signal is clearly stronger in the brighter atomic planes in the corresponding HAADF-STEM image $\left[{ }^{124}\right]$. 

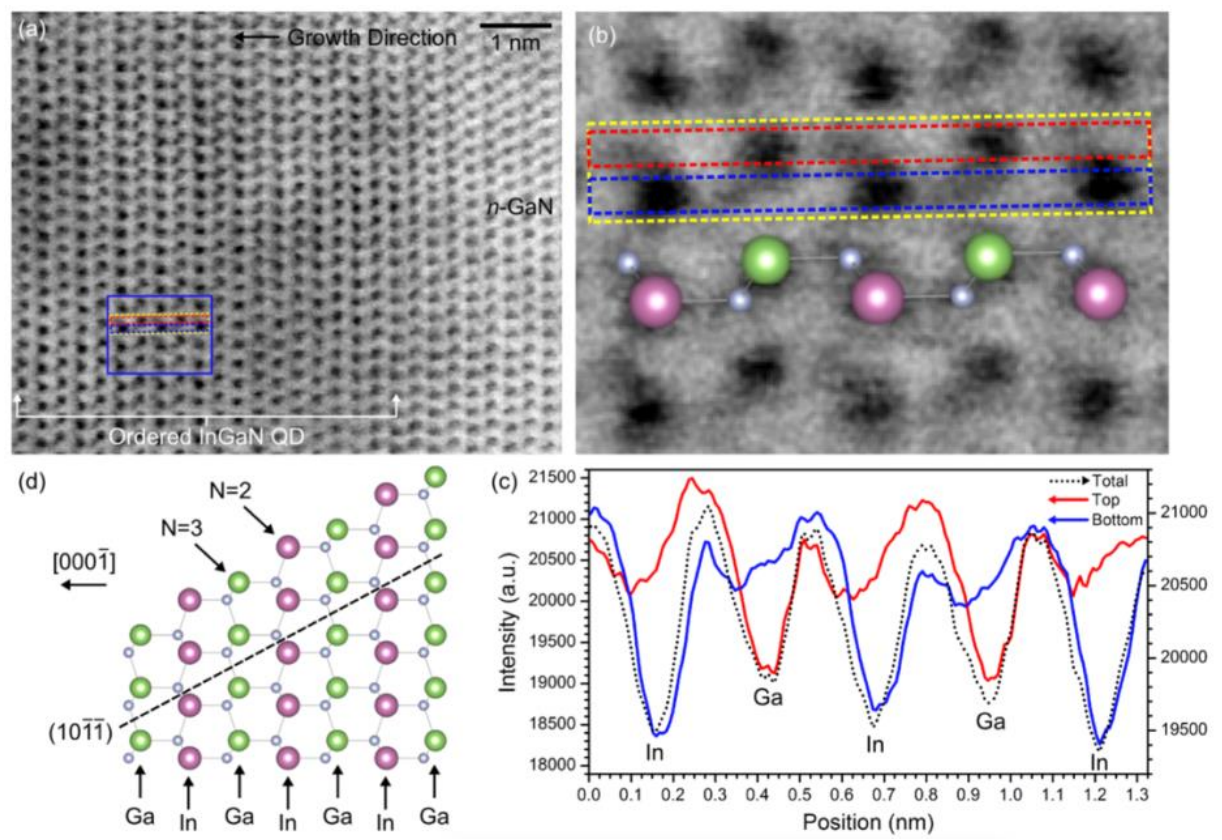

Fig. 20. Atomic ordering of GaN and InN in an InGaN quantum dot imbedded in a GaN nanowire. Reprinted from [124] with the permission of ACS

In summary, although aberration-corrected STEM imaging is a highly reliable method for providing evidence of atomic ordering in semiconductors, yet it has not been extensively used for the 1D nanostructures. Correlation of the atomic ordering with the physical properties of the ternary and quaternary semiconductor nanowires, can answer several questions regarding their functionality in devices such as solar cells and LEDs.

\subsubsection{Quantum structures}

The same STEM methodology has been employed to identify the presence of quantum structures within nanostructure semiconductors with atomic precision. It has been reported that ternary alloys used as nanowire shells, tend to exhibit a special segregation through which some parts of the shell, typically the corners and/or interfaces with the primary material, become richer in concentration of a certain element. This phenomenon, which mostly occurs for strain relaxation reasons, gives rise to formation of quantum structures within the nanowires. For identification of such segregation, cross-sectional STEM has been employed as an appropriate method, since it eliminates the effect of coverage caused by the outer structure, and provides a reasonable insight to the 'inner morphology' of the material. Realization of such structural details are of high importance since they influence the physical, especially optoelectronic, properties of the nanowires. Examples of GaAs-AllnP $\left[{ }^{125}\right]$, and GaAs-AIGaAs core-shell nanowires $\left[{ }^{126},{ }^{127}\right]$ have been reported.

In conventional STEM imaging, due to the electron probe size and presence of spherical aberrations, we can only reach the atomic resolution for the structures that have relatively large lattice parameters. The EDX/EELS spectroscopic signals in each pixel can also contain information of the surrounding area, because of the same technical reason, in addition to the proximity effect, meaning electron scattering in the specimen, giving information from an area larger than the probe size. For characterization of quantum structures, however, atomic resolution is essential, because the properties exhibited by them is highly dependent on their structural and chemical arrangement at the atomic scale. Hence, aberration-corrected STEM imaging and spectroscopy techniques should be employed. 
Several researchers have successfully produced intentional OD, 1D and 2D quantum structures which can be useful for various applications such as quantum photonics [ $\left.{ }^{128}\right]$. Review papers on this topic has been fairly recently published $\left[{ }^{129},{ }^{130}\right]$. Therefore, here we only point out a few highlights.

The first example is the GaAs-AIGaAs which has a wide range of applications in electronic and optoelectronic devices. GaAs-AlGaAs (multi-) quantum wells have been grown by different nanowire growth techniques, MBE and MOCVD [126, $\left.{ }^{131}, 132,133\right]$. Fig. 21 shows HAADF-STEM images on the cross-section (sliced by microtome method) of a GaAs-AlGaAs core-shell structure with a GaAs quantum well which is covered with a thin shell of AlGaAs and finally another shell of GaAs. It has been observed that these nanowires reveal a particular three-fold (instead of a six-fold) symmetry. The side facets of the nanowires are non-polar $\{110\}$ facets. At the corners (junctions of the two $\{110\}$ facets) small semi-polar $\{112\}$ facets also emerge which are responsible for this three-fold symmetry, since three of them are Apolar and the other three are B-polar. It is shown that the $\{112\} \mathrm{B}$ lateral facets are larger than their A-polar counterparts, and the Al-rich lines formed along them are considerably thicker. A thickness inhomogeneity in the GaAs quantum well is also notable, where it is thinner on $\{112\} B$ side and thicker on the A-polar side. It is worth pointing out that a key point in this study is determination of the polarity, since in the cross-sectional view of the nanowire, it cannot be determined. Therefore, the specimen requires to be tilted $35.5^{\circ}$ in order to reach a $<110>$ zone axis, from which the Ga-As dumbbell units can be visualized [126].

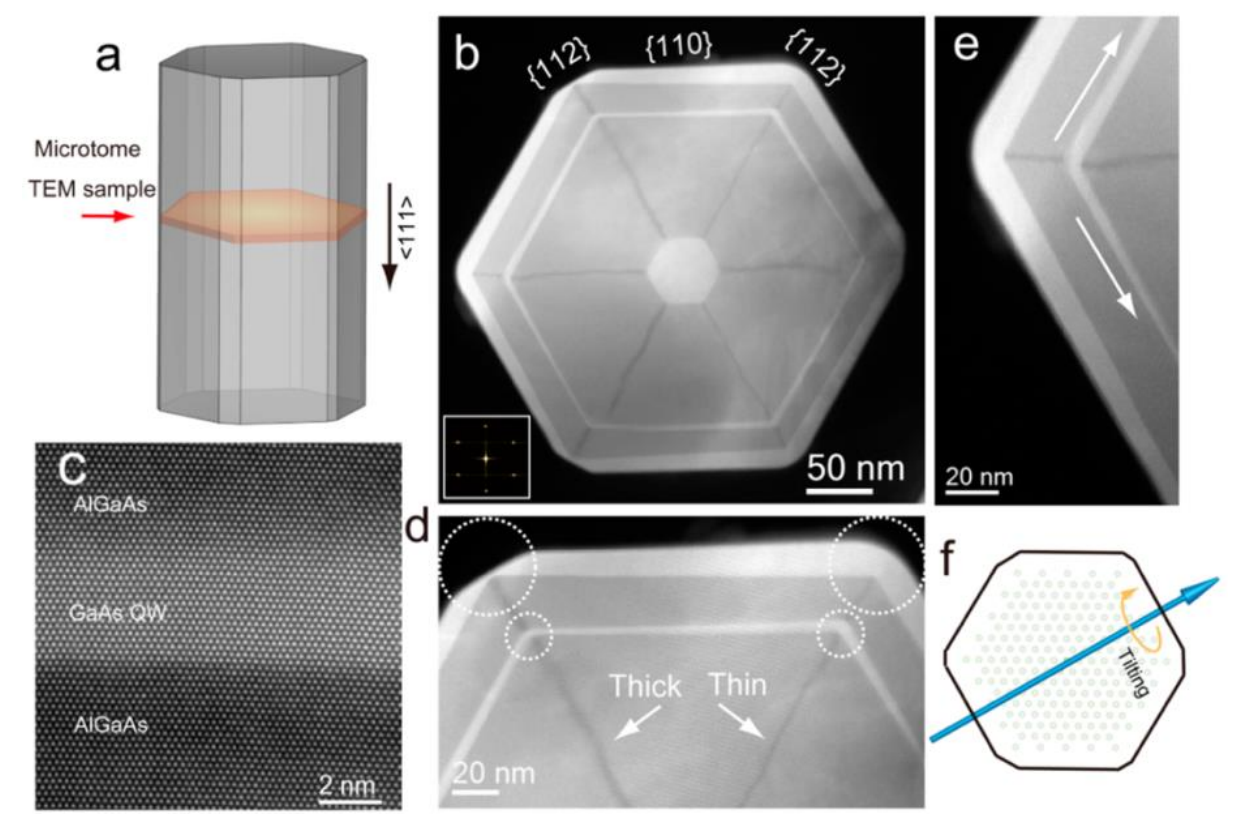

Fig. 21. Cross-sectional HAADF-STEM analysis of a GaAs-AIGaAs radial heterostructure, (a) schematic illustration of microtome-prepared slice, (b-d) HAADF-STEM images showing the three-fold symmetry of the nanowire in (b) lateral facets, (d) thickness of the Al-rich corners, and (e) thickness of the GaAs quantum well, (f) illustration of tilting direction toward a $<111>$ zone axis. Reprinted from [126] with the permission of ACS

Another example is the case of self-assembled GaN quantum wires in between an AIN core and shell forming at the corners. The width of these quantum wires, as deduced from the higher intensity in the atomic-resolution HAADF-STEM images in Fig. 22, is 1-2 atomic bilayers, and they only appear at the corners, filling up the truncation of the AIN core structure $\left[{ }^{134}, 135\right]$. 

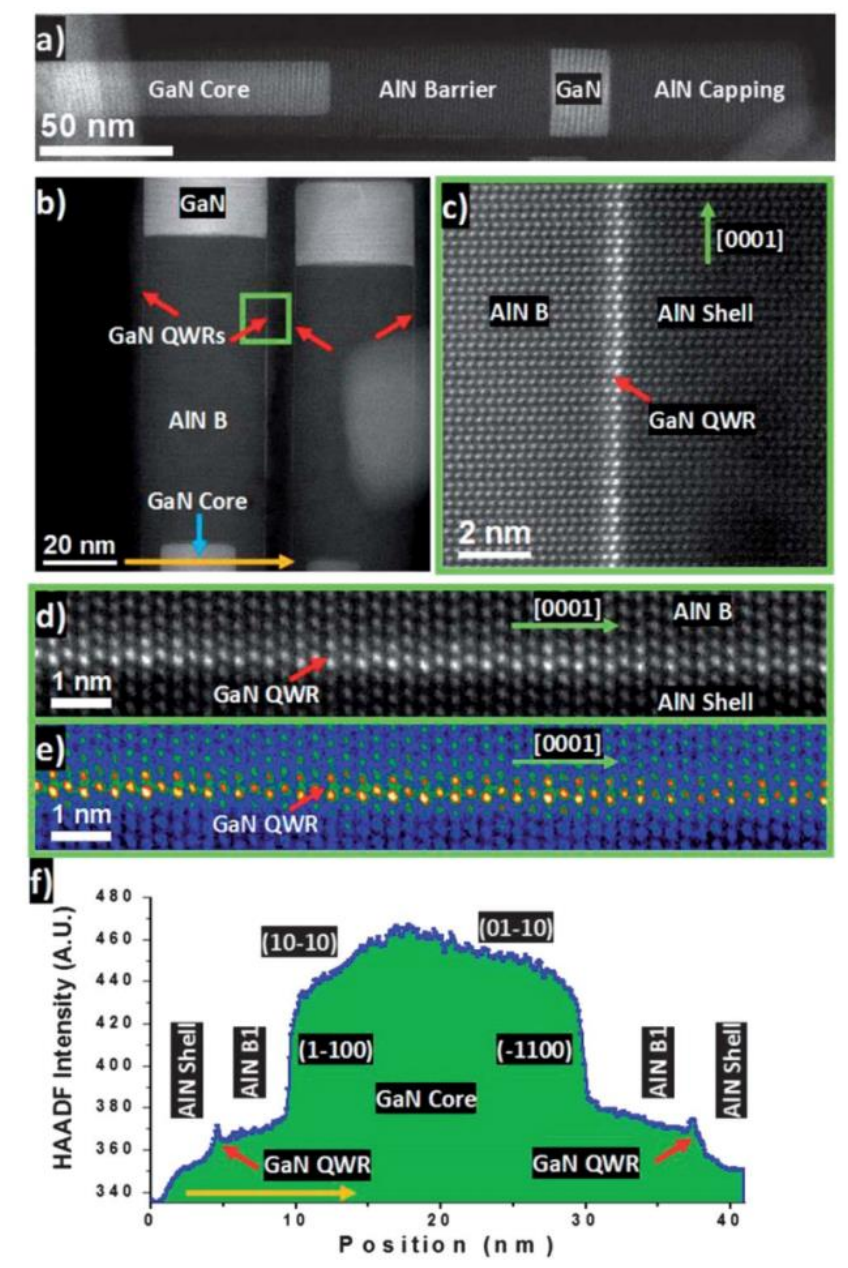

Fig. 22. HAADF-STEM images of the GaN-AIN heterostructure nanowires, (a) General overview, (b) GaN quantum wires indicated by red arrows, (c-e) atomic-resolution HAADF-STEM image of the GaN quantum wire; the brighter columns contain $\mathrm{Ga}$, (f) intensity profile along the orange arrow in (b). Reprinted from [134] with the permission of RSC

In 1D heterostructure nanowires, it is also important to take into account the abruptness of the transition from one structure to another. Intermixing of the two compounds, whether beneficial or detrimental, needs to be under control. This will be the topic of the next section.

\subsubsection{Heterojunctions}

Heterointerfaces in nanostructure semiconductor materials play an increasingly important role, especially in devices such as lasers, photovoltaics and transistors. Heterojunction engineering in such materials has shown particular promise since its freedom is increased due to the dislocation-free lateral strain relaxation $\left[{ }^{136}\right]$. The properties of the heterojunctions, determined by the local atomic arrangement at the junction, have a critical and direct impact on the behavior of the semiconductor devices. Therefore, there is a demand for availability of reliable and accurate characterization methods at the atomic scale. STEM imaging and spectroscopy techniques, in this context, have proven promising in providing crucial information on the structural and chemical aspects of the heterojunctions with atomic spatial resolution [72].

Here we present examples of different methods to distinguish the heterointerfaces in heterostructure nanowires. Spectroscopy methods, EDX and EELS, can provide precise 
information about the interfaces. In addition, ABF- and HAADF-STEM imaging have proven useful for identifying the atomic arrangement, by relative intensities of the atomic columns.

Conventional (S)TEM methodology has been used in order to obtain information at the nanoscale. There are several examples in the literature using intensity profile analysis and/or EDX/EELS in order to roughly show the sharpness of the heterointerfaces between different III-V structures such as axial GaAs-InAs [136], axial InAs-InP [137], InGaAs-InGaAs core-shell [138], GaAs-GalnP core-shell [ $\left.{ }^{139}\right]$, InAs-AlSb-InAs core-multi-shell $\left[{ }^{140}\right]$, axial Si-Ge $\left[{ }^{141},{ }^{142}\right]$, ZnTe-ZnSe core-shell [ ${ }^{143}$ ], and axial CdTe segments in ZnTe nanowires [144].

With the recent increasing interest towards achieving defect-free atomically-sharp heterointerfaces, aberration-corrected STEM has become more commonly employed, since atomic arrangement at the heterointerface can be directly and unambiguously visualized $[145,146,147,148]$. By controlling the sharpness of the heterointerfaces, one can tailor the electronic band alignment, and carrier distribution in the material, and hence the physical properties of them in the devices. Some methods have been proposed to tweak the growth procedure in a way that either the diffusion is suppressed, or the reservoir effect is reduced $[147,148]$.

Fig. 23 presents an Au-seeded MOVPE-grown InP nanowire with three InAs axial segments. $A$ very low growth temperature is used in order to achieve sharp axial heterointerfaces between InP and InAs. From the low-magnification HAADF-STEM images, Fig. 23(a,b), the InAs segments are clearly distinguishable and separated by their higher intensity, as well as their EDX signals. The properties of the heterointerfaces, however, can only be observed through atomic-resolution HAADF-STEM images, Fig. 23(c-e). It is observed that the transition from InAs to InP is different from the one of InP to InAs. The former exhibits a compositionally graded layer, where 3-4 atomic bilayers of a ternary InAsP forms (Fig. 23(c)). In contrast, the reverse transition, InP to InAs seems to be completely abrupt with no intermixing of the two compounds, as shown in Fig. 23(d). This heterointerface is further magnified in Fig. 23(e), where the dumbbell units can be resolved. Since $P$ is a light element, its atomic columns cannot be seen beside the ones of In as a heavy element. However, as the nanowires are conceivably B-polar (InAs dumbbells prove it) we can assume that the P atoms are on top in the dumbbell units, as shown by green circles in Fig. 23(e). In this image the atomically sharp heterointerface from InP to InAs is clearly evident. Such high controllability of the heterointerfaces, as well as the size of the InAs segments enables tailoring of the luminescence properties of the nanowires [147].

(a)

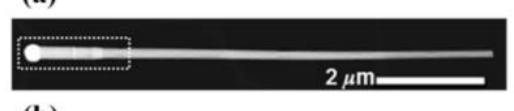

(b)

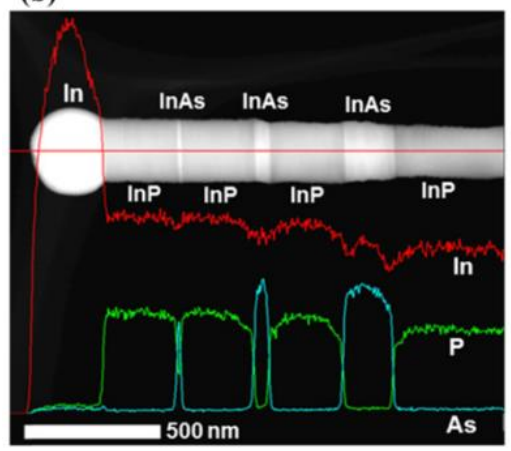

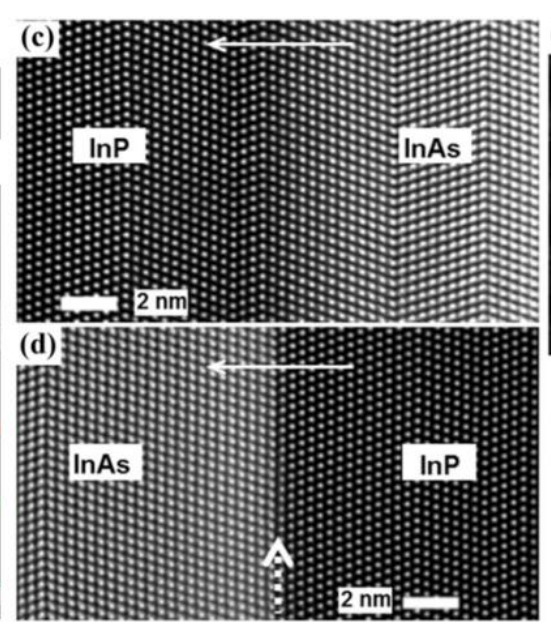

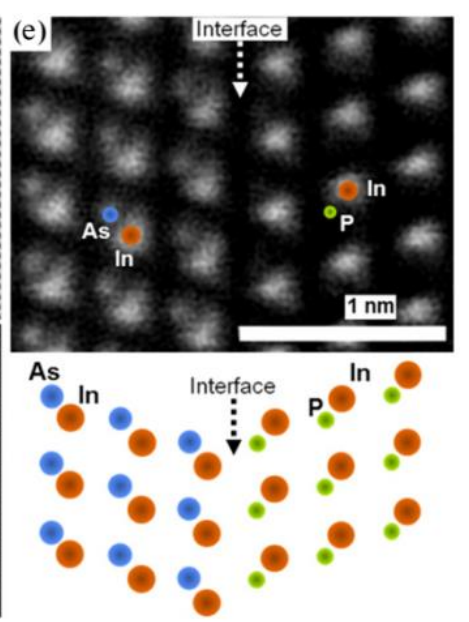

Fig. 23. HAADF-STEM images of an InP nanowire containing InAs axial segments,

(a) overview of the nanowire, (b) position of the InAs segments shown both by 
Z-contrast and EDX signals of In, P and As, (c,d) atomic-resolution HAADF-STEM images of the axial (c) $\ln A s$ to $\ln P$, and (d) $\ln P$ to $\ln A$ s heterointerfaces showing that the latter is sharper; (e) the exact position of the atomically sharp InP to InAs heterointerface together with atomic structure. Reprinted from [147] with the permission of IOP

The axial heterointerfaces of the aforementioned are formed via vapor-liquid-solid (VLS) growth mechanism. On the other hand, the radial interfaces grow via vapor-solid (VS) mechanism since no liquid particle is involved, and we only have absorption of atoms from the gas phase. The VS growth occurs either via step-growth, diffusion of atoms from the substrate, or birth and spread, direct nucleation on the side facets and spreading from there. Therefore, the reservoir effect is absent.

As another example, Fig. 24 shows a wurtzite InAs-GaSb core-shell nanowire. In fact, wurtzite GaSb is not stable in bulk, and its realization in the form of nanowire shell (via crystal structure transfer method) demands extreme growth conditions, i.e. higher temperatures and molar fractions. In low-magnification HAADF-STEM image of Fig. 24(b), for InAs-GaSb the intensity should vary only with the thickness, since the sum of atomic numbers for InAs and GaSb (as compounds) are exactly the same, as well as their crystal structure (both are wurtzite), and their crystal lattice parameters are quite close. However, the radial heterointerface between the InAs core and GaSb shell appears slightly darker than the rest. This can suggest that lighter elements are contributing to the heterointerface. In order to clarify this hypothesis, atomic-resolution HAADF-STEM imaging, together with postexperiment image filtering (deconvolution of the probe effect) and simulation [44] is performed, Fig. 24(c). The intensity profiles obtained from the dumbbell units are shown in Fig. 24(d): GaSb shell in green, heterointerface in purple, and InAs core in blue. The relative intensities of the dumbbells of the core and the shell are as expected, up to the closest ones to the heterointerface. This reveals an almost abrupt transition, except for the one single atomic bilayer at the heterointerface. At the heterointerface, the intensities of the dumbbell atoms are similar to the ones of the lighter elements, i.e. Ga and As at the position of the group III and V atoms, respectively. However, the intensity of the group III element appears slightly higher than the one of group $\mathrm{V}$, which should not be the case as $\mathrm{Ga}$ is slightly lighter than As. Therefore, it is conceived that In is also incorporating in the heterointerface structure, forming one atomic bilayer of a ternary InGaAs compound. Having two consecutive IDBs does not seem feasible, since their formation is not energetically favorable. In the same study, Namazi et al have shown that the reverse transition from GaSb to InAs has identical properties. The same InGaAs ternary material emerges at the GaSb-InAs radial heterointerface in wurtzite InAs-GaSb-InAs core-shell-shell structure [71]. 


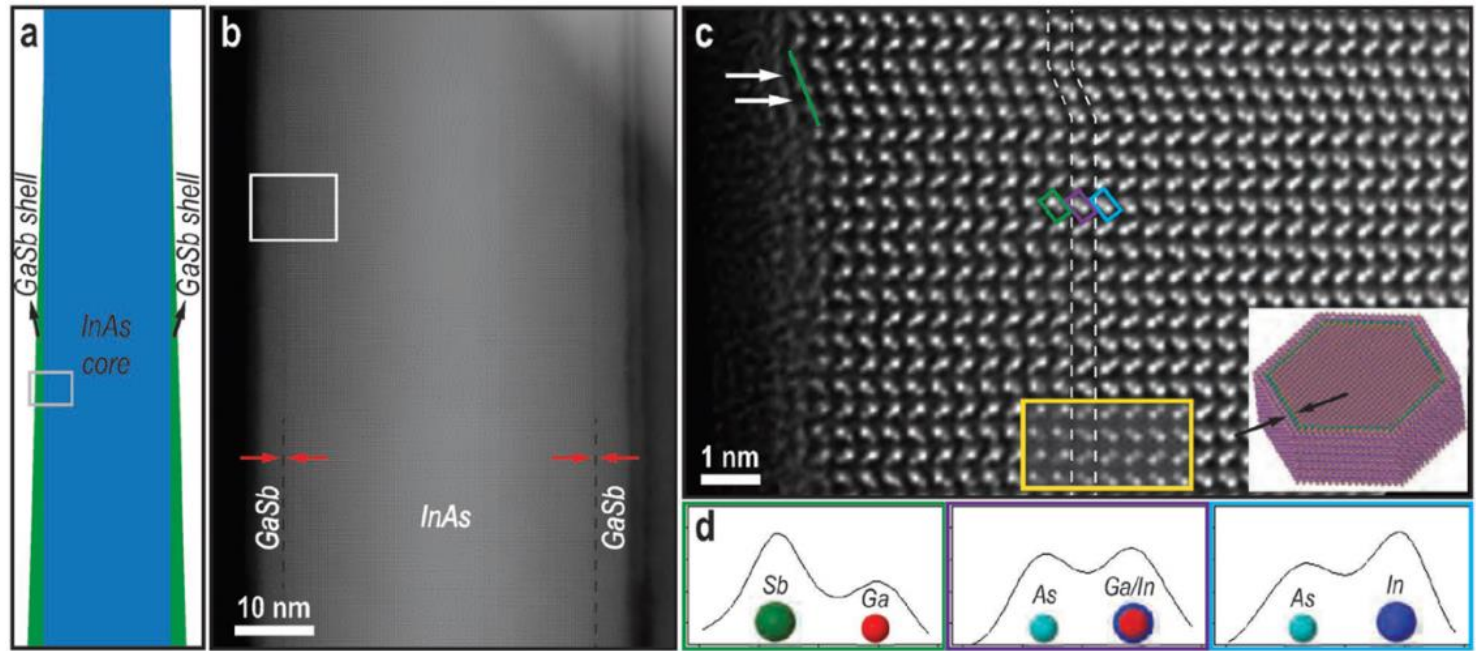

Fig. 24. HAADF-STEM images of an InAs-GaSb core-shell nanowire, (a) schematic and (b) overview of the radial part of nanowire, $(c)$ atomic-resolution HAADF-STEM image (after deconvolution) of the radial heterointerface showing one single atomic bilayer of a ternary InGaAs compound; (d) intensity profiles of the indicated dumbbell units of InAs shell (green), GaSb core (blue), and InGaAs ternary compound at the heterointerface (purple). Reprinted from [71] with the permission of Wiley

In the last example we present a radial/axial zinc-blende GaSb-InAs heterostructure nanowire system, where the interfaces have been identified by atomic-resolution spectrum imaging of core-loss EELS chemical signals. The difference with the previous example is that the material system is reversed, meaning GaSb is the core and InAs is the shell, and more importantly, here we have zinc-blende structure instead of wurtzite. The main difference, which is important for observation of the radial heterointerfaces, is that in wurtzite nanowires, the planes of radial heterointerfaces, $\{1-100\}$ side facets, are along the $\langle 1-100\rangle$ zone axis and can clearly be observed. For zinc-blende nanowires, however, the heterointerfaces are along the six $\{110\}$ planes, and none of them have the facet normal perpendicular to the zone axis. Therefore, what we see at the heterointerface is in fact a corner resulting from the junction of two $\{110\}$ planes. Atomic-resolution EELS maps, nevertheless, provide the accurate information on these heterointerfaces [72].

The GaSb-InAs radial heterostructure is shown in Fig. 25(a) and the interface is magnified in Fig. 25(b) to show the atomic columns. The white box indicates the region on which the EELS maps are acquired. Fig. 25(c-i) show the maps of the EELS extracted signals (Fig. 25(j-m)). By overlaying each two signals, Ga with Sb (Fig. 25(g)), In with As (Fig. 25(h)), the dumbbell units are reconstructed, and by overlaying all four signals (Fig. 25(i)), we can locate the heterointerface precisely. From these maps it can be reliably deduced that the radial heterointerface is atomically sharp. Counting out the delocalization effect of the EELS signal which is quite weak in such high energy losses, no Ga or Sb signals can be detected on the right side of the maps. The weak signals of In and As detected on the left side (GaSb region), nevertheless, come from the InAs shell that is covering the core. Note that quantitative interpretation of atomically resolved EELS data is much more complicated due to the complex beam propagation and dynamic effects [72]. 

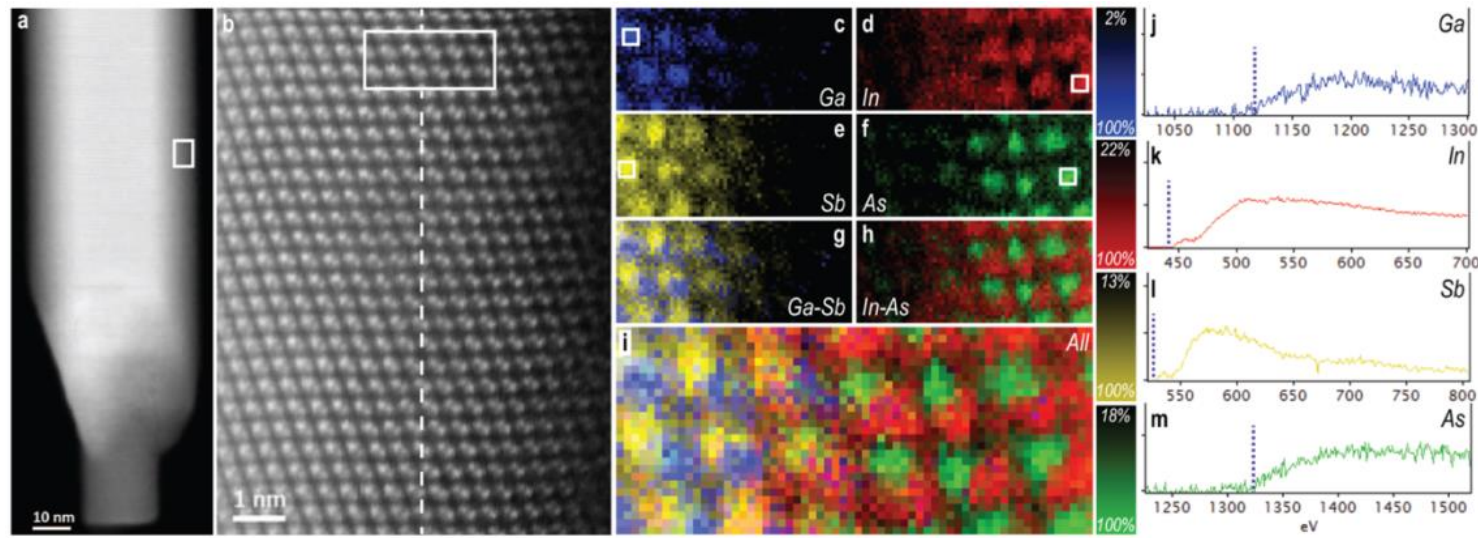

Fig. 25. Atomic-resolution EELS spectrum imaging on GaSb-InAs radial heterointerface, (a) low-magnification and (b) atomic-resolution HAADF-STEM images of the radial part of the nanowire, (c-i) EELS elemental maps at the atomically-sharp radial heterointerface, $(\mathrm{j}-\mathrm{m})$ extracted EELS signals of the four elements. Reprinted from [72] with the permission of ACS

The axial heterointerface, in contrast to the radial one, reveals an interfacial region with the two compounds intermixed for a few atomic bilayers. This can be clearly observed in the EELS maps in Fig. 26. The dash lines in Fig. 26(b) indicate the position of the heterointerfaces, and the white box marks the region from which the EELS maps are acquired. This region is intentionally chosen close to the lateral facets since such regions are thinner and provide better clarity and reliability of the extracted chemical maps. At this region, some atomically localized signals of Ga and Sb (Fig. 26(c) and Fig. 26(e), respectively) can be observed above the nominal interface (dash line), which become weaker moving upward in the InAs segment. This suggests that $\mathrm{Sb}$ is intermixed with InAs, which is attributed to the reservoir effect, i.e. presence of the residual $\mathrm{Ga}$ and $\mathrm{Sb}$ atoms in the Au seed particle. On the other hand, strong signals of In and As (stronger for As) is detected in the GaSb part. This suggests that In and As atoms diffuse downwards during the growth. It should be noted that this signal is stronger that the contribution of the InAs shell around GaSb, since it decreases dramatically by moving away from the interfacial region [72].
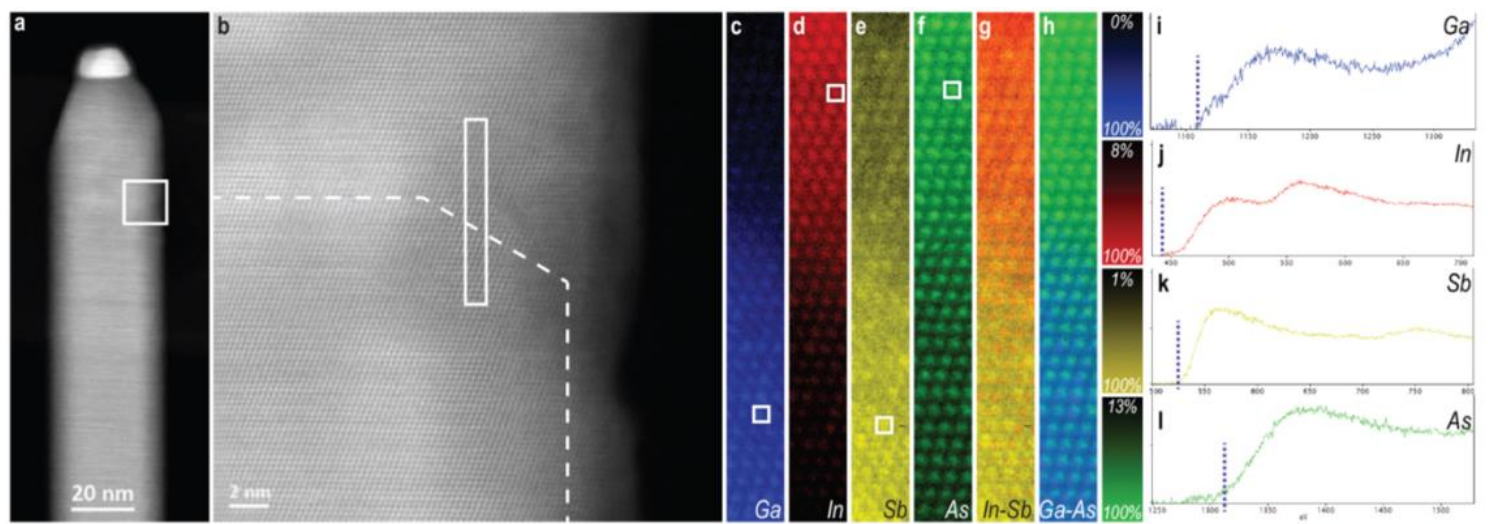

Fig. 26. Atomic-resolution EELS spectrum imaging on GaSb-InAs axial heterointerface, (a) low-magnification and (b) atomic-resolution HAADF-STEM images of the axial part of the nanowire, (c-h) EELS elemental maps at the axial heterointerface revealing the intermixing and gradual change from GaSb to InAs, ( $i-I)$ extracted EELS signals of the four elements. Reprinted from [72] with the permission of ACS 
In summary, understanding the heterointerfaces at the atomic scale is of high importance, especially when such materials systems are used as semiconductor devices. As shown in the examples of this section, they can have dramatic influence on the physical, e.g. electronic and optoelectronic, properties of the material system.

\subsubsection{Lattice strain}

The impact of lattice strain on a wide range of material properties, has been extensively studied. Nanostructured materials, however, respond to strain differently from their bulk counterparts. It is even shown that by strain engineering, one can tune the optical and electronic properties of such materials at will $\left[51,{ }^{149}, 150,151\right]$. For instance, it has been shown that by employing both compressive and tensile strain on GaAs-AlGaAs-GaAs core-shell nanowires, one can tune and boost their optoelectronic properties, and reduce their bandgap [150]. Strain engineering can be achieved by either mechanical manipulation or creating heterostructures. Calculation of strain on bare 1D nanostructure materials is relatively straight forward. On the other hand, such materials have a unique ability to accommodate secondary structures with relatively higher mismatches (in comparison with their bulk counterparts) and compensate for strain. This is due to their free-standing geometry that they can laterally relax the strain from the growth direction instead of causing emergence of misfit dislocations. In this section we mention examples of strain studies caused by mechanical manipulation, as well as the growth of heteroepitaxial structures.

We start off with an example of strain engineering and measurement performed by a novel method of in-situ mechanical micromanipulation and electrical measurement in TEM, in combination with STEM-NBED for mapping the strain field. It has been employed on Auseeded MBE-grown InAs-InGaAs core-shell nanowires. The 4-nm-thin InGaAs shell is only used to passivate the InAs surface in order to avoid surface states that can cause errors in the electrical measurement. The mechanical forces are employed by means of a nanoindenter holder with an electrical push-to-pull microelectromechanical system. Then, in STEM mode the electron beam scans the whole nanowire, and at each pixel, the NBED pattern is recorded. The axial and radial strains are calculated by measuring the displacements of the (0002) and (11-20) diffraction spots, respectively, with respect to the non-strained spot positions [51].

Strain in crystal lattice alters charge carrier transport properties of the material by modifying the band structure and charge carrier scattering characteristics. Therefore, the I-V curves are recorded simultaneous with tensile force being applied by the nanoindenter holder, in order to correlate the strain with the electrical properties of the nanowire. Strain maps, $\varepsilon_{x x}, \varepsilon_{y y}$, and rotational strain, are shown in Fig. 27(a-c). In Fig. 27(d) the average strains, $\varepsilon_{x x}$ and $\varepsilon_{y y}$, are plotted versus tensile stress applied by the nanoindenter holder. The I-V curves, shown in Fig. 27(e), firstly reveal the Ohmic contact behavior since they are linear at both ends. Secondly, their linearity and symmetry suggest that there is minimum piezoelectric effect; and more importantly, they show that the resistance of the nanowire decreases continuously with the tensile stress. Such direct correlation of the electrical properties of the semiconductor nanowires with lattice strain caused by mechanical stress, is only possible by employing the combination of an in-situ micromechanical manipulator together with NBED in STEM mode [51]. 


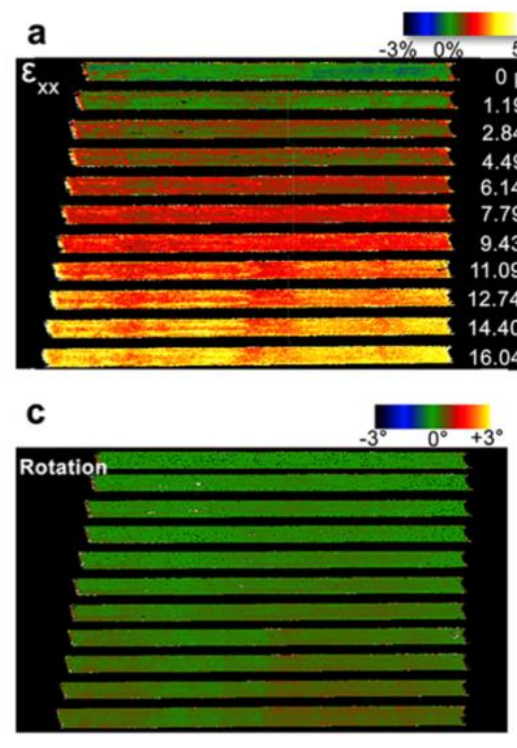

b
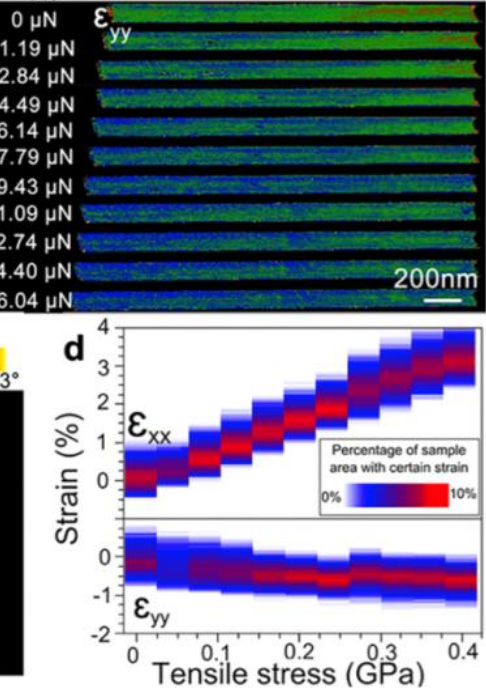

e
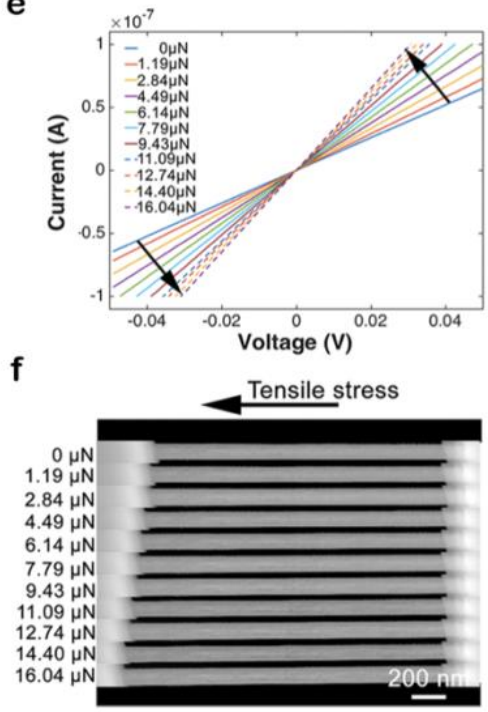

Fig. 27. Strain distribution in an InAs-InGaAs core-shell nanowire under in-situ tensile stress; lattice strain distribution maps (a) along the nanowire, (b) perpendicular to the nanowire, and (c) the corresponding rotational maps at different tensile stress; (d) quantitative strain versus tensile stress; (e) I-V curves characteristics of the nanowire under tensile stress, (f) corresponding HAADF-STEM images of the nanowire under tensile stress. Reprinted from [51] with the permission of ACS

An alternative method for strain field mapping is the geometric phase analysis (GPA). The sensitivity of GPA is directly dependent on the resolution and quality of the high-resolution (S)TEM images on which the method is applied. An advantage of GPA is the possibility of strain mapping at the atomic scale. For this reason, it has been extensively used in semiconductor nanowires, especially at the heterointerfaces, revealing the elastic or plastic strain field (with presence or absence of misfit dislocations $[69,152,153,154,155]$.

Fig. 28 proposes an atomic scale strain relaxation mechanism in an axial wurtzite GaAs zinc-blende GaSb heterostructure nanowire. Theoretical mismatch between the lattice parameters of wurtzite GaAs and zinc-blende GaSb is $7.8 \%$ in the growth direction. Atomicresolution HAADF-STEM images reveal a phase transition at the axial interface, where the GaAs structure changes from wurtzite to zinc-blende, and after a few atomic bilayers the interface between zinc-blende GaAs and zinc-blende GaSb occurs. This latter interface, unlike the former, is not flat. It has a curvature (or a truncation) that presumably occurs due to changes in the configurations of the Au seed particle, because of either surface energy differences during the switching sequence or a change of its volume resulting to wetting of the sides. By performing GPA at these interfaces and the region around (Fig. 28(e,f)), it is observed that at the GaAs-GaSb bent heterointerface, periodic misfit dislocations emerge on average every $4.8 \mathrm{~nm}$. Through occurrence of misfit dislocations, 7.6\% dilatation takes place at the heterointerface according to GPA strain maps, which compensate for almost all the strain. Regarding the elastic strain component, a small rotation at the edges of the nanowire is detected, $+1.5^{\circ}$ on the left edge and $-3^{\circ}$ on the right (Fig. $28(f)$ ). The difference comes from the presence and absence of misfit dislocations. On the left edge, two misfit dislocations can be observed, which almost compensate for the elastic strain. On the other hand, no dislocations are detected on the right. Therefore, larger rotations occur [69]. 


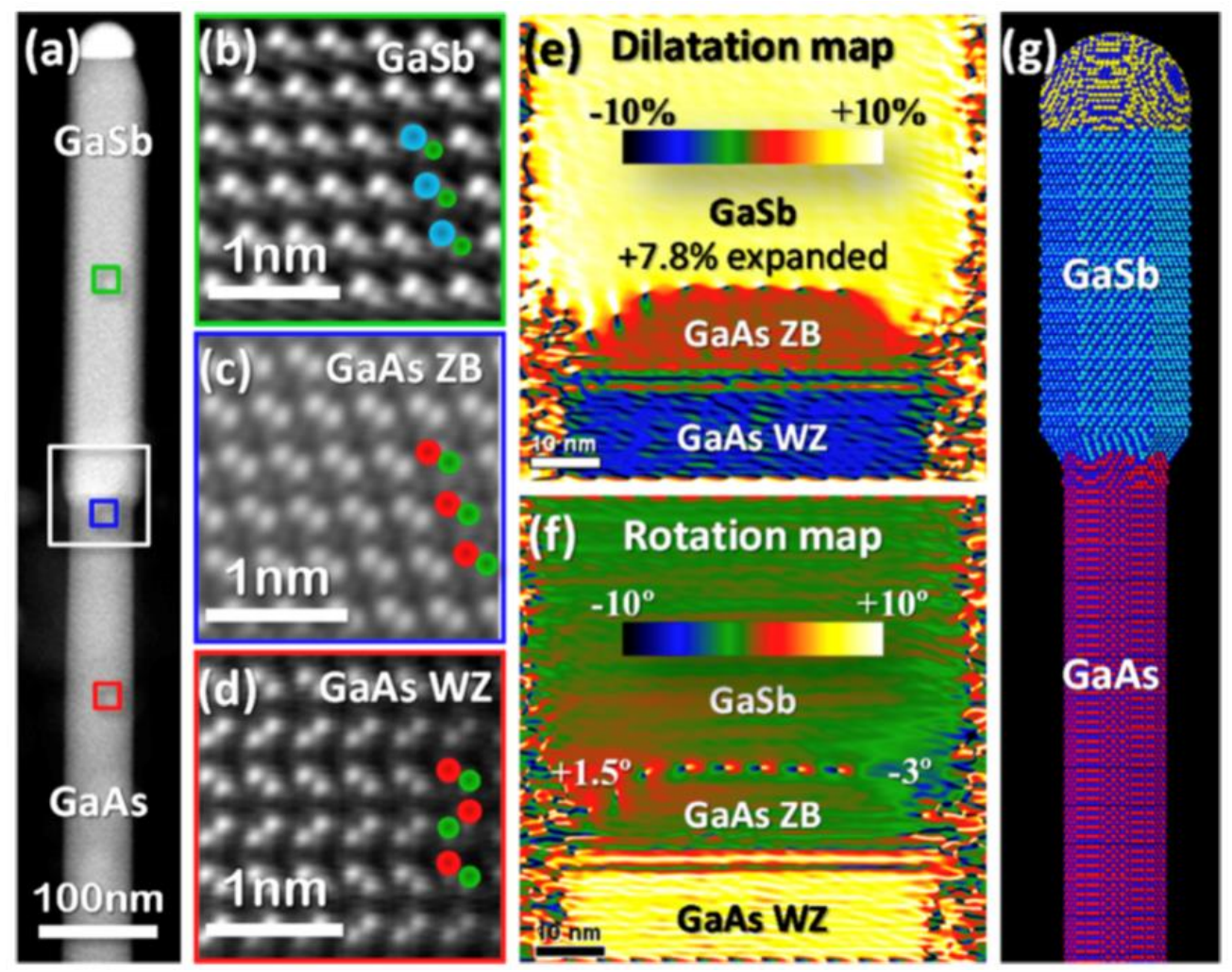

Fig. xxv. GPA at the axial heterointerface of wurtzite GaAs and zinc-blende GaSb; (a) overview HAADF-STEM image, (b-d) atomic-resolution HAADF-STEM images of the (b) zinc-blende GaSb, (c) zinc-Blende GaAs at the axial heterointerface, and (d) wurtzite GaAs; (e) dilatation and ( $f$ ) rotational strain maps at the heterointerface, (g) 3D atomic model of the heterostructure nanowire. Reprinted from [69] with the permission of ACS

The detailed strain analyses are especially important in the newly developed selected area or surface guided growth of nanowires. New developments in nanodevices for future applications such as quantum computing, need a perfect epitaxy of the semiconductor components with the substrate in order to facilitate perfect circuit connections avoiding grain boundaries and/or poor contacts. In this way, new technologies make use of the nanoengineering in order to create nanowire networks attached to the substrates. This new playground which is different from the typical VLS vertical nanowire growth, implies new actors in the game, namely the substrate. Relaxation mechanisms between the substrate and the nanowires $\left[143,{ }^{156},{ }^{157}\right]$ or nanomembranes $\left[{ }^{158},{ }^{159}\right]$ grown on top of those substrates and buffers, are nowadays being investigated in detail at the atomic scale to minimize the formation of misfit dislocations and other detrimental defects such as inversion domains.

\subsection{Physical properties}

As shown in the previous section, the ample range of (S)TEM imaging and spectroscopy techniques can potentially be used to resolve several structural aspects of the materials, especially 1D nanostructure semiconductors. The capabilities of (S)TEM methodology, nevertheless, goes far beyond structural properties. In this section we discuss its potentials for obtaining information on the physical properties of the semiconductors via three topics: First, measurement of optical properties, in particular the bandgap, by means of low-loss EELS; second, measurement of luminescence properties of wider bandgap semiconductors by STEM-CL; and finally measurement of internal electric fields at the atomic level, by DPC imaging. 


\subsubsection{Optical properties by EELS}

Electronic bandgap alignment in nanostructure semiconductors is an important characteristic which determines their electronic and optical properties. By tuning the bandgap one can modulate the properties of the nanostructure semiconductors and use them for desired applications. This requires a suitable method for measuring the bandgap with high accuracy, and sufficient spatial resolution, in order to understand the complex arrangements, when it comes to heterostructures, especially $p-n$ nanojunctions. Among the currently available techniques, photoluminescence $(\mathrm{PL})$ is the most common which provides information on optical bandgap of the semiconductors $\left[{ }^{160}\right]$. It can be combined with SEM for localized measurements. Although it has high accuracy in energy scale, it does not provide sufficient spatial resolution in order to observe the band structure in different parts of a complex heterostructure nanomaterial. Valence EELS (VEELS), on the other hand, is a promising technique with which we can measure the bandgap with very high spatial resolution of few nanometers.

VEELS has been used to determine the bandgap of semiconductors such as GaN and InGaN with different In contents $\left[{ }^{161}, 162,163\right]$. It is also employed to show the chemical composition variation in interfaces of heterostructure layered semiconductor materials such as GaAsInGaAs $\left[{ }^{164}\right]$ and grain boundaries in $\mathrm{Cu}(\mathrm{In}, \mathrm{Ga}) \mathrm{Se}_{2}\left[{ }^{165}\right]$. Such variations result in changes in the bandgap along the interface. Furthermore, VEELS allowed to identify modifications in the local density of states in different GaN polytypes, revealing changes in the band to band electronic transition energy from $3.4 \mathrm{eV}$ for pure wurtzite $\mathrm{GaN}$ to $3.2 \mathrm{eV}$ in zinc-blende-like twinned lattice regions consisting on just 3 cubic monolayers, within the same GaN nanowire [163]. More recently, it has been shown by VEELS that the different polytypes of SiC nanowires have different bandgaps, as shown in Fig. 29. The bandgap of wurtzite $\mathrm{SiC}$ is measured to be $3.3 \mathrm{eV}$ while its Zinc-blende polytype exhibits narrower bandgap of $2.5 \mathrm{eV}$. This can be used as a signature to detect the structural defects along the nanowires. It is shown that the adjacent stacking faults do not affect the bandgap of the wurtzite regions. For the zinc-blende parts, in contrast, the bandgap is influenced by the thickness of the segments [ $\left.{ }^{166}\right]$.

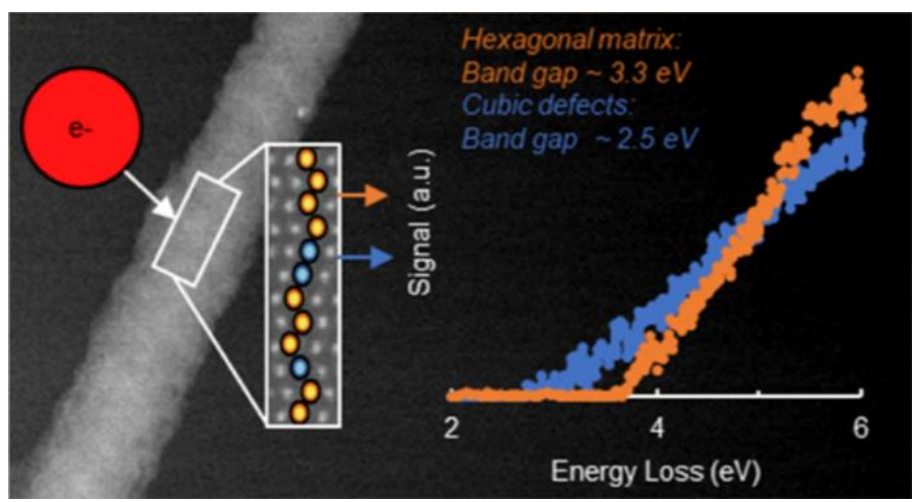

Fig. 29. VEELS on polytypic SiC nanowires, revealing the difference in the change of band gap energy from hexagonal matrix to cubic defects. Reprinted from [166] with the permission of ACS

In another study, this method is used for determination of the bandgap of MOCVD-grown $\mathrm{In}_{\mathrm{x}} \mathrm{Ga}_{1-\mathrm{x}} \mathrm{N}$ nanowires with various In content in the full range of $\mathrm{x}=0$ to 1 . Here the bandgaps are measured and compared by three different methods: Optical absorption, PL, and VEELS. In Fig. 30(left), the morphology of the grown nanowires with different In contents is shown. 
The samples are ordered by their In content, from sample 1, pure GaN, to sample 13, pure $\operatorname{InN}$. The plot on the right is the collection and comparison of all the results obtained from PL emission spectra, optical absorption spectra, and VEELS measurements. All the measurement methods seem to be in good agreement with one another, and match the expected value of the bandgap (Bowing fit) within a reasonable error $\left[{ }^{167}\right]$.
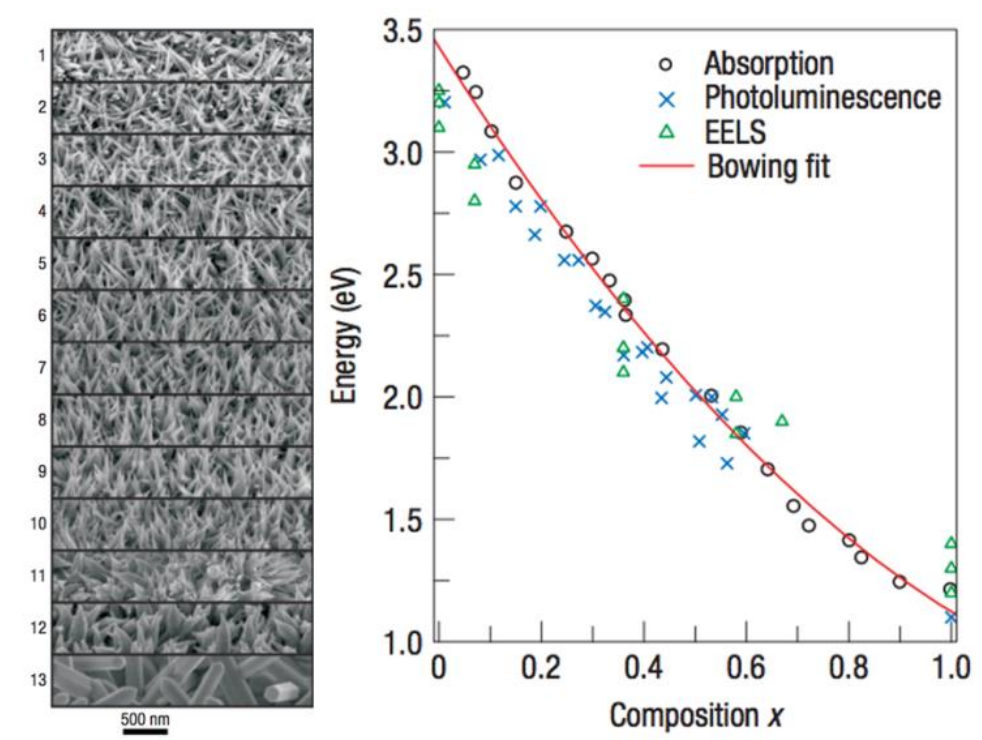

Fig. 30. Band gap energy of $\ln _{x} G_{1-x} N$ nanowires versus their In content, measured by three methods: absorption, photoluminescence, and VEELS. Reprinted from [167] with the permission of Nature

The use of this technique for nanowires is challenging since there are issues with characterization due to drift and stability. However, since the nanowires do not require destructive specimen preparation methods (such as FIB) and they can simply be transferred to the carbon grids (for instance by mechanical proximity method), their structure will remain undamaged. This increases the reliability of the acquired data. Moreover, their diameter, commonly between 10 to $120 \mathrm{~nm}$, makes them suitable for such measurements. Therefore, this method has a great, and yet unexplored, potential to be used in the field of 1D nanostructured semiconductors.

\subsubsection{Luminescence properties via $\mathrm{CL}$}

Cathodoluminescence $(C L)$ is another technique with great potential to explore the optical properties of the semiconductors. SEM-CL is more commonly used for this aim. There are several studies in the literature using this technique to measure the optical properties $\left[{ }^{168}\right]$, for different purposes such as examining the effect of chemical modifications such as nitridation $\left[{ }^{169}\right]$, determining the doping level $\left[{ }^{170}\right]$, observing the effect of stacking sequence within the structure [171], and polarity [67]. It can also be used to study the carrier concentration [ $\left.{ }^{172}\right]$.

STEM-CL has a better spatial resolution, and it can be coupled with high-resolution STEM imaging and/or chemical mapping methods such as EELS or EDX [173]. Since this Review is about the advanced applications of STEM-based techniques, we only mention some recent examples of STEM-CL. The recently published Review by Kociak and Zagonel gives a full overview of the technique and its applications for nanostructured materials [ $\left.{ }^{174}\right]$. Here we only point out a few recent examples of STEM-CL studies on III-V (including nitrides) nanowires.

It is well-known that the presence of stacking faults in semiconductors affect their optical properties, usually by hindering the luminescence. However, in the Ga-polar GaN nanocolumn 
shown in Fig. 31, the intensity in the panchromatic $\mathrm{CL}$ emission increases in the vicinity of the lower two defects indicated by the arrows. A line scan is also performed along the length of the nanocolumn. The luminescence data is shown in Fig. 31(c). It can be observed that in the base of the nanocolumn the near-band-edge emission is dominant. Closer to the stacking faults the near-band-edge emission disappears, and instead, an additional higher emission appears which is the result of generated excess carriers that have diffused from the stacking faults. This higher emission is in fact due to the formation of a quantum well of a zinc-blende structure (as a result of twinning in wurtzite). In addition, a slight red shift occurs at the tip of the nanocolumn, which is speculated to be due to either polarization and strain, or termination of the stacking fault with the semi-polar facets of the tip [ $\left.{ }^{175}\right]$.
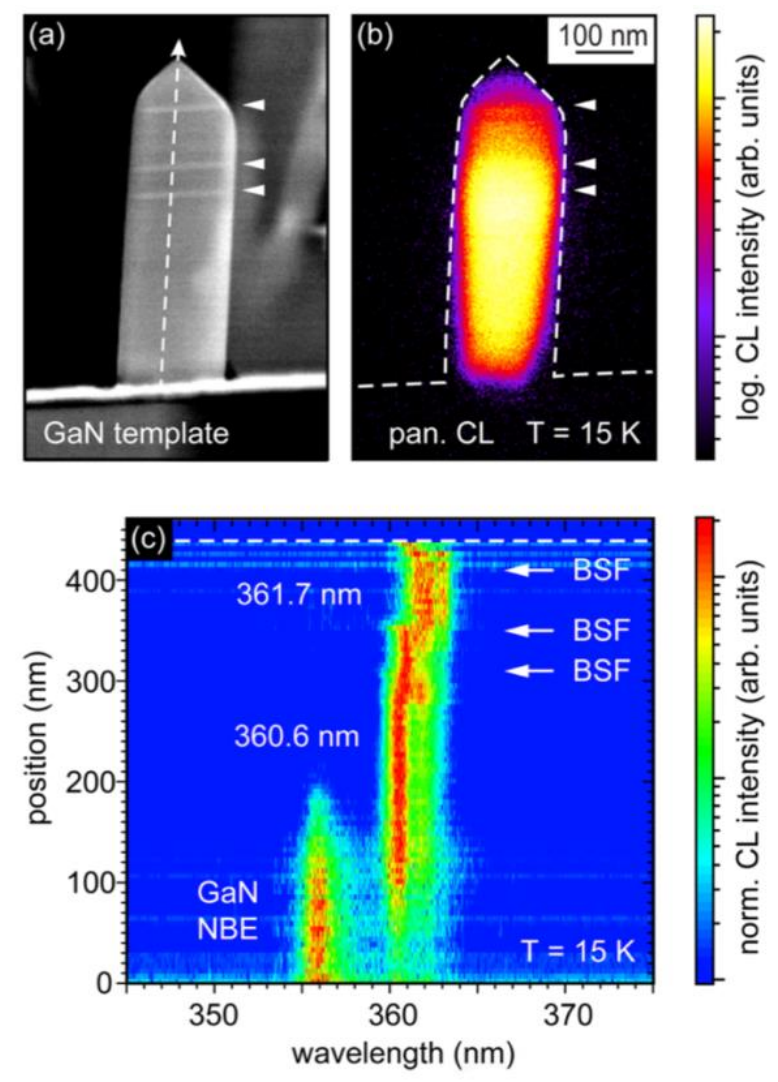

Fig. 31. (a) HAADF-STEM image of a GaN nanocolumn, containing three stacking faults as indicated, (b) panchromatic $C L$ intensity image showing the highest intensity at the two lower defects, (c) CL spectrum along the nanocolumn (dash line arrow). Reprinted from [175] with the permission of ACS

In another study, again by Christen and co-workers, STEM-CL is performed on a heterostructure Ga-polar GaN nanorod, consisting of a Si-doped GaN core with a thin AIGaN layer separating it from the Si-doped GaN shell. Thereafter, a single InGaN quantum well is deposited on this shell, and it is covered with a final undoped GaN layer. Direct visualization of the optical properties of this complex structure is shown in the low-temperature STEM-CL maps in Fig. 32. CL intensity map in Fig. 32(a) reveals that the strongest luminescence comes from the InGaN quantum well formed on the non-polar facets, while the weakest is detected from the Si-doped GaN core, which is most probably due to the increased density of point defects. Fig. 32(b) is the color-coded CL peak wavelength map and Fig. 32(c) shows the normalized spectra obtained from the indicated regions of the nanorod. Yellow luminescence is dominant in the lower part of the GaN core, due to Ga vacancies and O complexes, and no 
near-band-edge emission is detected. Unlike the core, the GaN shell exhibits dominantly the donor-bound exciton emission, indicating a high optical quality. AlGaN layer also shows a different wavelength peak at the semi-polar facet. There is also a clear energy shift of InGaN emission along the non-polar side facet, together with the peak broadening, which can be caused by localized effects of the generated carriers. Moreover, the emission of the non-polar InGaN quantum well is observed to be different from the one on the semi-polar facet. In summary, the CL signal can be obtained with high spatial resolutions, giving a clear insight to the optical properties of complex heterostructure semiconductors [ $\left.{ }^{176}\right]$.

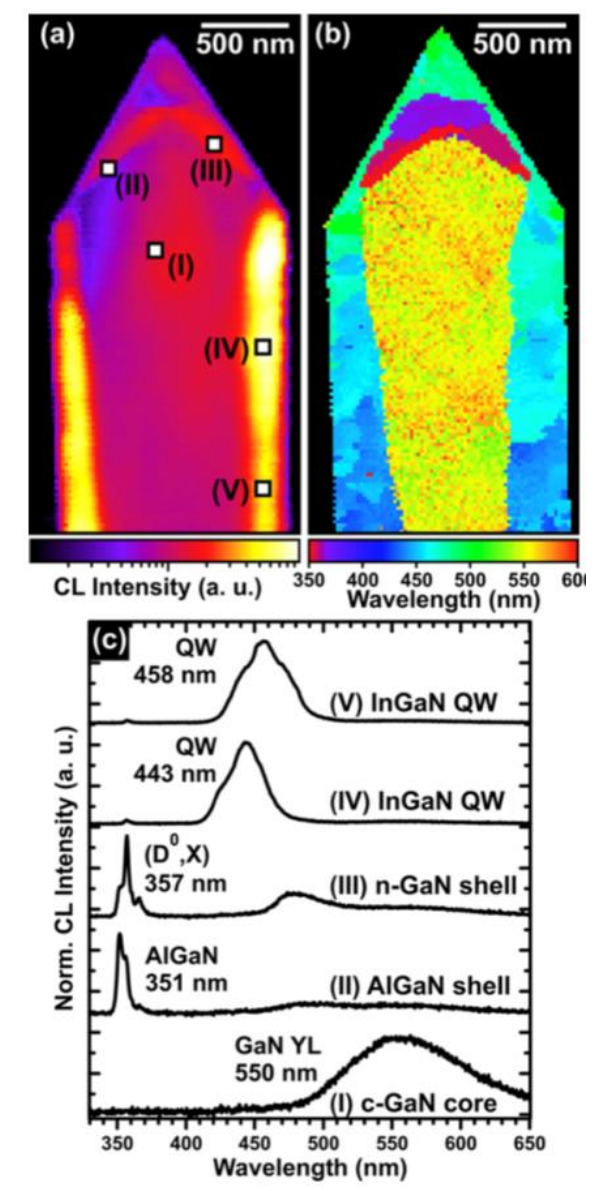

Fig. 32. (a) Panchromatic CL intensity image on a GaN-AlGan-InGaN heterostructure nanocolumn, (b) color-coded CL peak wavelength image, (c) $\mathrm{CL}$ spectra obtained on different parts of the nanocolumn as indicated in (a). Reprinted from [176] with the permission of ACS

Beside the emission energy, the lifetime is an important parameter for the electron-hole states. Both of these parameters can vary depending on several factors, such as confinement potential due to the size in quantum structures, presence of electric fields, presence of defects, or surface states. The last example of this section, is the study of the emission lifetime of atomically thin GaN quantum discs in an AIN nanowire matrix, by means of the novel method of spatially-resolved time-correlated (SRTC)-CL [177].

The decay of photon emission can form photon bunches when they are related to the lower energy states, such as in defects where the electron-hole pairs are diffused to. This arises in a time window of their lifetime $(\tau)$, which is typically in the nanosecond range. This bunching behavior is seen as a peak of high amplitude in the second order correlation function, $g^{(2)}(\tau)$, which decays exponentially with the emitter's lifetime [177]. 
Fig. 33 shows the HAADF-STEM image of eight GaN quantum discs imbedded in an AIN nanowire, in correlation with the SRTC-CL spectra. There is a space of $15 \mathrm{~nm}$ between each of the GaN quantum discs, which makes their emissions distinguishable. Fig. 33(b) is the colorcoded spectral map of the nanowire, where each energy layer has a different color. It shows that the emissions in the quantum discs are not uniform, which is due to thickness variations and strain effects. Lifetimes of the quantum discs, measured by SRTC-CL, are shown in Fig. 33(c). Interestingly, longer lifetimes correspond to lower emission energies, and shorter ones to higher energies. This is an expected behavior from such strongly polar material. In the presence of a spontaneous electric field, the electrons and holes tend to separate and become localized on the different sides of the quantum discs. This separation increases with the width of the quantum discs and thus the emission energy decreases. As a result, smaller energies correspond to reduced electron-hole spatial overlaps and increased lifetime [177].
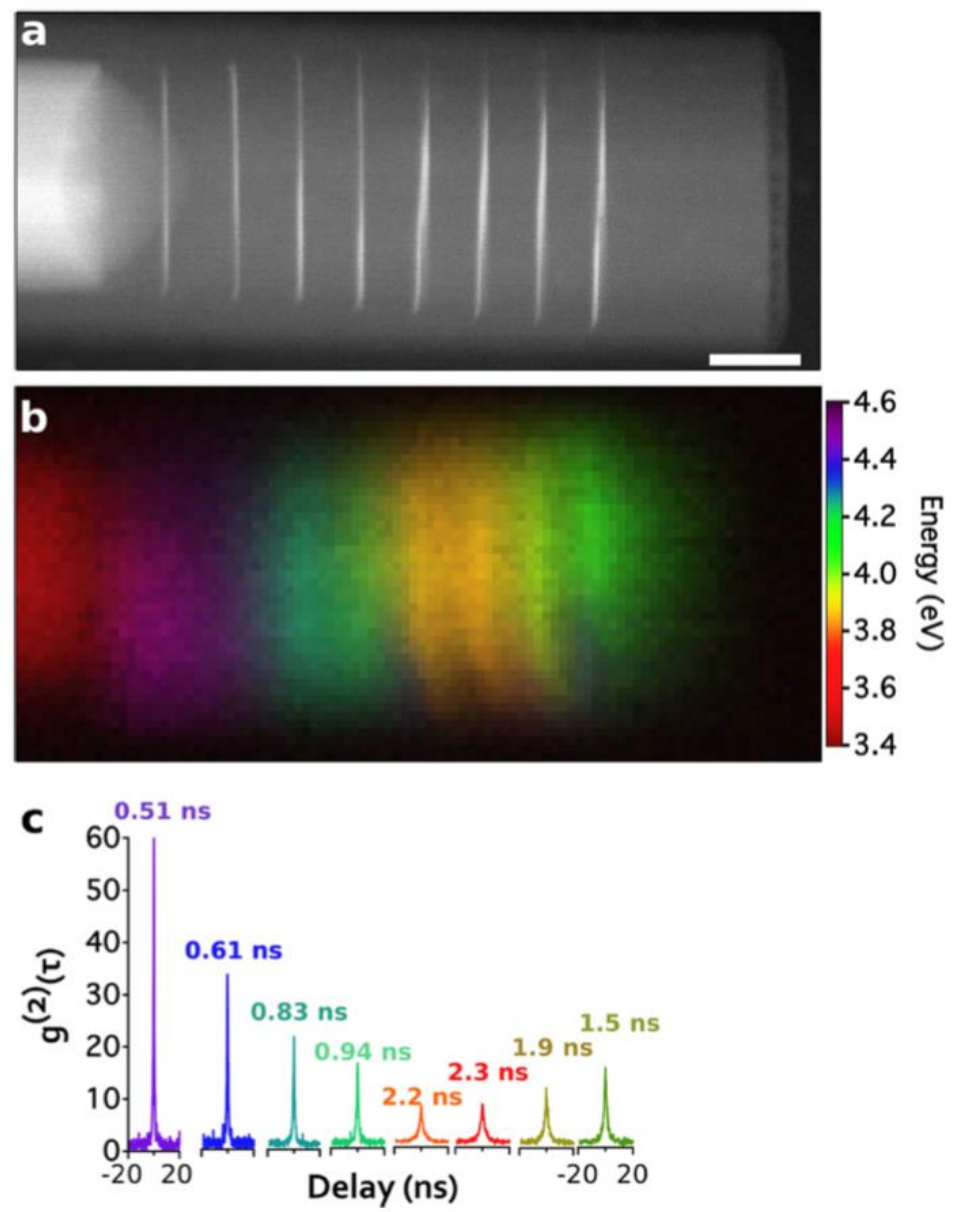

Fig. 33. SRTC-CL on GaN quantum discs in an AIN nanowire, (a) HAADF-STEM image of the quantum dics, in correlation with (b) color-coded CL spectral map, (c) emission lifetime of in each quantum disc. Reprinted from [177] with the permission of ACS

Mentioning the internal electric field in the polar materials leads us to the final section which is measurement of these fields by means of DPC imaging, as a recently developed STEM imaging technique with high potentials for both magnetic materials and semiconductors.

\subsubsection{Electric field via DPC imaging}

Differential phase contrast (DPC) imaging in STEM is a powerful method for characterization of the electric fields at the atomic scale. As described in Section 2.1 this can be achieved by employing segmented or pixelated STEM detectors. Moreover, it can provide 
mesoscopic information about the polarization fields in the domains of the material. This method, to the best of our knowledge, has not yet been employed to semiconductor nanowires. However, it has a great potential to derive this field towards novel device applications. Here we only mention a very recent example of this method employed on GaN 2D materials [ $\left.{ }^{178}\right]$.

Fig. 34(a) schematically shows the interaction of the electron probe with a GaN atomic column and the detection of the signal by the segmented detector. As can be seen, the probe intensity at the diffraction plane is shifted toward the Ga column. This is due to the interaction of the electron probe with the electron field of the Ga atoms. Fig. 34(b) shows the HAADFSTEM image of the region. As expected, $\mathrm{N}$ atomic columns cannot be seen since $\mathrm{N}$ is a light element in comparison with Ga. The orthogonal components of the center of mass of the intensity distribution at the diffraction plane (I $\mathrm{CoM}$ ), shown in Fig. 34(c), are obtained by weighting the signals acquired in each of the detector segments. The black contrast indicates an upward/leftward shift of the center of the mass, and white contrast, the opposite. This can be more clearly observed in the colored electric field vector map in Fig. 34(d). This image shows that the electric fields point outward from the nucleus. The magnitude of these electric fields, as seen in Fig. 34(e), shows that they are considerably stronger around the Ga atomic columns in comparison to $\mathrm{N}$ ones [178].

With a thin specimen with the thickness precisely measured, DPC imaging has the potential of quantitative mapping of the projected total charge density distribution. It can even discern subatomic details since it can visualize both the positive charge of the nucleus and the negative charge of the electrons in real space [178]. Therefore, as mentioned, this method has potential applications for studying the semiconductor nanowires.
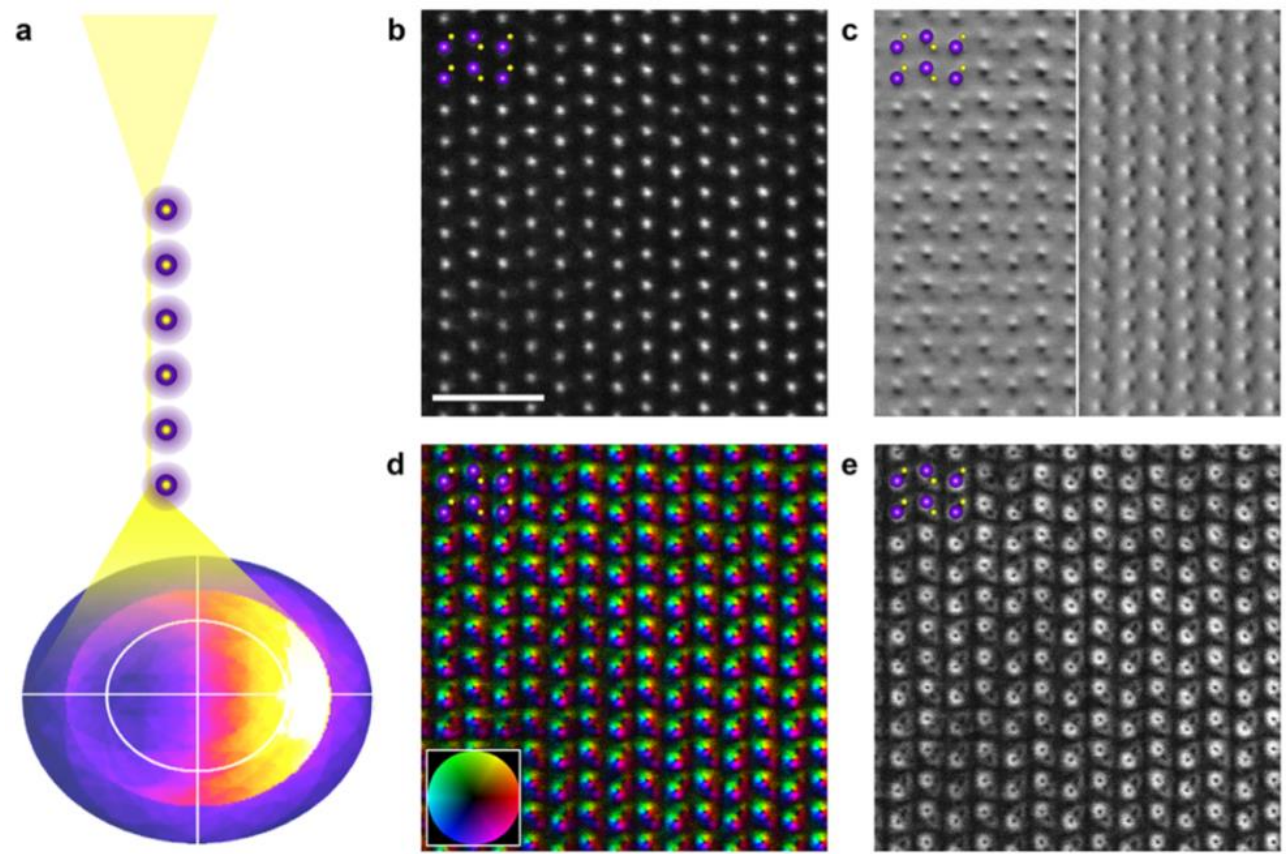

Fig. 34. DPC imaging on GaN, (a) schematic of interaction of the electron probe with GaN atomc column, (b) atomic-resolution HAADF-STEM image of GaN, (c) orthogonal components of the center of mass of the intensity distribution at the diffraction plane ( $\left.\mathrm{I}_{\mathrm{CoM}}\right)$, (d) colored electric field vector map, (e) magnitude of electric fields. Reprinted from [178] with the permission of ACS 


\section{Conclusion}

In summary, the wide and versatile range of STEM techniques, has dramatically expanded the borders of our knowledge of semiconductors in the last decade. This was not even imaginable without the technological and conceptual advancements of (S)TEM. Nowadays, having a state-of-the-art (S)TEM device in hand, one can obtain various information of different aspects of a material, from structural and chemical issues, to optical and luminescence properties, to electric and magnetic fields at the atomic scale with high precision. Most of the abovementioned techniques are nowadays being employed for characterization of novel nanostructure semiconductor systems, in order to understand their mechanical, optical and electronic properties as components of the newly-developed semiconductor devices. Several examples have been mentioned in this Review in order to show the possibilities that we have in hand to study our novel semiconductor materials and tailor their properties at will. Yet there is plenty of room to explore more by employing the novel STEM methodology and use the obtained knowledge and information to further improve the functionality and efficiency of the semiconductor devices.

\section{Acknowledgements}

RRZ acknowledges the Chalmers Materials Analysis Laboratory (CMAL). JA acknowledge funding from Generalitat de Catalunya 2017 SGR 327. ICN2 acknowledges support from the Severo Ochoa Programme (MINECO, Grant no. SEV2013-0295) and is funded by the CERCA Programme / Generalitat de Catalunya.

\section{References}

\footnotetext{
1 AR Verma, Nature 167 (1951) 939

2 FC Frank, Adv. in Phys. 1 (1952) 91-109

${ }^{3}$ S Conesa-Boj et al, Cryst. Growth Des. 10 (2010) 1017-1020

${ }^{4}$ SPT Svensson et al, Nanotechnology 19 (2008) 305201

${ }^{5} \mathrm{~F}$ Qian et al, Nano Lett. 5 (2005) 2287-2291

${ }^{6}$ W Guo et al, Nano Lett. 10 (2010) 3355-3359

7 JA Czaban et al, Nano Lett. 9 (2009) 148-154

$8 \mathrm{~J}$ Ho et al ACS Photonics 2 (2015) 165-171

9 D Saxena et al, Nano Lett. 16 (2016) 5080-5086

${ }^{10} \mathrm{E}$ Lind et al, Nano Lett. 6 (2006) 1842-1846

${ }^{11} \mathrm{M}$ Haider et al, Ultramicroscopy 75 (1998) 53-60

12 M Haider et al, Nature 392 (1998) 769-770

${ }^{13} \mathrm{HH}$ Rose, J. Electron Microscopy 58 (2009) 77-85

${ }^{14}$ C Hetherington, Materials Today 7 (2004) 50-55

${ }^{15} \mathrm{M}$ de la Mata et al, Nano Lett. 12 (2012) 2579

16 J Zuniga-Perez et al, Appl. Phys. Reviews 3 (2016) 041303

${ }^{17}$ X Yuan et al, Adv. Mater. 27 (2015) 6096-6103

${ }^{18}$ B Freitag et al, Ultramicroscopy 102 (2005) 209-214

${ }^{19}$ D Jacobsson et al, Nature 531 (2016) 317

${ }^{20}$ Y-C Chou et al, Science 343 (2014) 281

21 J-C Harmand et al, Phys. Rev. Lett. 121 (2018) 166101

22 MI den Hertog, Nano Lett. 9 (2009) 3837-3843

${ }^{23}$ S Yazdi et al, Small 11 (2015) 2687-2695

${ }^{24}$ S Lehmann et al, Nanotechnology 26 (2015) 301001
} 
${ }^{25}$ M Perillat-Merceroz et al, Nanotechnology 23 (2012) 125702

${ }^{26} \mathrm{~V}$ Zannier, et al., Nano Lett. 16 (2016) 7183

27 J David, et al., Nano Lett. 17 (2017) 2336

${ }^{28}$ L.M. Brown, J. Phys. F: Metal Phys. 11 (1981) 1-26

${ }^{29}$ Aberration-Corrected Analytical Transmission Electron Microscopy, SuperSTEM book Ch3

${ }^{30}$ WW Mathews, Trans. Am. Microsc. Soc. 2, 190-195 (1953)

${ }^{31}$ Dinges, C., Kohl, H. \& Rose, H. Ultramicroscopy 55, 91-100 (1994)

${ }^{32}$ R Ishikawa et al, Nat. Mater. 10 (2011) 278

${ }^{33} \mathrm{M}$ de la Mata, J Arbiol, book chapter, High Resolution in STEM mode (2013) 375-425

${ }^{34} \mathrm{~N}$ Shibata et al, J Electron Microscopy, 59 (2010) 473-479

${ }^{35} \mathrm{M}$. Krajnak et al, Ultramicroscopy 165 (2016) 42-50

${ }^{36}$ TJ Pennycook et al, Ultramicroscopy 151 (2015) 160-167

${ }^{37} \mathrm{H}$ Yang et al, Nat. Comm. 7 (2016) 12532

${ }^{38} \mathrm{~S}$ Gao et al, Nat. Comm. 8 (2017) 163

${ }^{39}$ N Shibata et al, Nat. Phys. 8 (2012) 611

${ }^{40}$ DS Gemmell, Rev. Modern. Phys. 46 (1974) 129-227

${ }^{41}$ P Stadelmann, www.jems-saas.ch

${ }^{42}$ C Koch, www.physics.hu-berlin.de/en/sem/software/software_qstem

${ }^{43}$ J Barthel, www.er-c.org

${ }^{44}$ V Grillo, E Rotunno, Ultramicroscopy 125 (2013) 97-111

${ }^{45}$ A Rosenauer and M Schowalter, Microscopy of Semiconducting Materials 120 (2008) 170-172

${ }^{46}$ A De Backer et al, Ultramicroscopy 171 (2016) 104-116

${ }^{47}$ EJ Kirkland et al, Ultramicroscopy 23 (1987) 77-96

${ }^{48} \mathrm{~S}$ Bernal et al, Ultramicroscopy 72 (1998) 135-164

${ }^{49}$ VB Ozdol et al, Appl. Phys. Lett. 106 (2015) 253107

${ }^{50}$ C Gammer et al, Appl. Phys. Lett. 109 (2016) 081906

${ }^{51}$ L Zeng et al, Nano Lett. 18 (2018) 4949-4956

52 MJ Hytch et al, Ultramicroscopy 74 (1998) 131-146

${ }^{53}$ AB Yankovick et al, Nat Comm 5 (2014) 4155

${ }^{54}$ T Nilsson Pingel et al, Nat Comm 9 (2018) 2722

${ }^{55}$ NJ Zaluzec, Microscopy \& Microanal. 20 (2014) 1318-1326

${ }^{56}$ VJ Keast et al, Phys. Rev. B 66 (2002) 125319

57 O Nicoletti et al, Nature 502 (2013) 80-84

${ }^{58}$ B Rafferty and LM Brown, Phys. Rev. B 58 (1998) 10326

${ }^{59}$ OL Krivanek et al, Nature 514 (2014) 209-212

${ }^{60} \mathrm{M}$ Stoger-Pollach et al, Micron 37 (2006) 396-402

${ }^{61}$ BG Yacobi, DB Holt, CL book 1990

${ }^{62}$ M Kociak, LF Zagonel, Ultramicroscopy 176 (2017) 112-131

63 JT Griffiths et al, Nano Lett. 15 (2015) 7637-7643

${ }^{64}$ LHG Tizei, M Kociak, Phys. Rev. Lett. 110 (2013) 153604

${ }^{65} \mathrm{~S}$ Meuret et al, ACS Photonics 3 (2016) 1157-1163

${ }^{66} \mathrm{~F}$ Schuster et al, ACS Nano 8 (2014) 4376-4384

67 M Zamani et al, Nanoscale 10 (2018) 17080-17091

${ }^{68} \mathrm{~F}$ Schuster et al, Nano Lett. 12 (2012) 2199-2204

${ }^{69} \mathrm{M}$ de la Mata et al, Nano Lett. 14 (2014) 6614-6620

$70 \mathrm{M}$ de la Mata et al, Nano Lett. 16 (2016) 825-833

${ }^{71} \mathrm{~L}$ Namazi et al, Adv. Func. Mater. 28 (2018) 1800512

72 RR Zamani et al, Nano Lett. 18 (2018) 1557-1563

${ }^{73} \mathrm{MI}$ den Hertog et al, Nano Lett. 12 (2012) 5691

${ }^{74}$ SD Carnevale et al, Nano Lett. 13 (2013) 3029

${ }^{75}$ A Urban et al, New J. Phys. 15 (2013) 053045

${ }^{76}$ MD Brubaker et al, Cryst. Growth \& Des. 16 (2016) 596-604

77 M Hetzl, Nano Lett. 17 (2017) 3582-3590

78 P Wang et al, Nano Lett. 16 (2016) 1328-1334

${ }^{79}$ B Zhao et al, Nanoscale 10 (2018) 11205

${ }^{80}$ MIB Utama et al Nano Lett. 12 (2012) 2146

${ }^{81} \mathrm{~L}$ Manna et al, Nat. Mater. 2 (2003) 382

82 DJ Milliron et al, Nature 430 (2004) 190-195

83 RR Zamani et al, ACS Nano 8 (2014)2290-2301

${ }^{84}$ MIB Utama et al Adv. Func. Mater. 23 (2013) 1636-1646 
85 E. Uccelli et al, Nano Lett. 11 (2011) 3827

${ }^{86}$ RR Zamani et al, Nanoscale 9 (2017) 3159-3168

87 D Car et al, Adv. Mater. 26 (2014) 4875

$88 \mathrm{~J}$-H Kang et al, Nano Lett. 13 (2013) 5190

89 J Wang et al, Nano Lett. 13 (2013) 3802

${ }^{90} \mathrm{HJ}$ Joyce et al, Nano Lett. 10 (2010) 908-915

${ }^{91} \mathrm{M}$ Ibanez et al, Chem. Mater. 24 (2012) 4615

92 PR Buseck, S lijima, Am. Mineralogist 70 (1975) 771-784

${ }_{93} \mathrm{H}$ Kohno et al, Cryst. Res. Tech. 38 (2003) 1082

${ }^{94} \mathrm{MI}$ den Hertog at al, Nanotechnology 23 (2011) 025701

${ }^{95} \mathrm{~S}$ Lehmann et al, Nano Lett. 13 (2013) 4099

${ }^{96} \mathrm{~S}$ Assali et al, Nano Lett. 15 (2015) 8062-8069

${ }^{97} \mathrm{H}$ Schmid et al, Micron 43 (2012) 49-56

${ }^{98}$ AP Goldstein, et al, ACS Nano 7 (2013) 10747-10751

${ }^{99}$ SC Andrews et al, Chem. Science 2 (2011) 706

$100 \mathrm{~J}$ Hoemke et al, J. Am. Ceramic Soc. 101 (2018) 2616-2626

$101 \mathrm{~J}$ Hoemke et al, J. Am. Ceramic Soc. 100 (2017) 4252-4262

102 M Dahl et al, Small (2018) 1703785

${ }^{103}$ AM Sanchez et al, Nano Lett. 17 (2017) 2454

${ }^{104}$ AM Sanchez et al Nano Lett. 18 (2018) 3081

$105 \mathrm{MI}$ den Hertog et al, Nano Lett. 8 (2008) 1544-1550

${ }^{106}$ G-S Park et al, Nano Lett. 12 (2012) 1638

${ }_{107}$ M Bar-Sadan et al, Nano Lett. 12 (2012) 2352

${ }^{108}$ R Senga et al, Nat. Mater. 13 (2014) 1050

109 TS Kuan et al Phys. Rev. Lett.54 (1985) 201

${ }^{110}$ SB Zhang et al, Phys. Rev. B 57 (1998) 9642

${ }^{111}$ Wei and Zunger, Phys. Rev. B 57 (1998) 8983

112 Detz and Strasser, J. Appl. Phys. 114 (2013) 123508

113 TM Christian et al, J. Appl. Phys. 114 (2013) 074505

${ }^{114} \mathrm{~K}$ Mukherjee et al, Appl. Phys. Lett. 106 (2015) 142109

${ }^{115}$ ET Yu et al, J. Vac. Sci. Tech. A 17 (1999) 2246

116 TS Kuan et al Phys. Rev. Lett. 54 (1985) 201

${ }_{117}$ H-M Wu et al, Thin Solid Films 570 (2014) 390-393

118 TM Christian et al, J. Appl. Phys. 114 (2013) 074505

119 Stringfellow \& Chen, J. Vac. Sci. Tech. B 9 (1991) 2182

${ }^{120}$ M Ibanez et al, Chem. Mat. 24 (2012) 562-570

${ }^{121}$ Esser et al, Phys. Rev. Lett. 117 (2016) 176101

${ }^{122} \mathrm{M}$ Wu et al, Appl. Phys. Lett. 105 (2014) 041602

${ }^{123}$ RR Zamani, PhD Thesis, University of Barcelona (2013)

${ }^{124}$ SY Woo et al, Nano Lett. 15 (2015) 6413-6418

$125 \mathrm{~N}$ skold et al, Nano Lett. 6 (2006) 2743-2747

${ }^{126}$ C Zheng et al, Nano Lett. 13 (2013) 3742-3748

127 D Rudolph et al, Nano Lett. 13 (2013) 1522-1527

${ }^{128} \mathrm{M}$ Heiss et al, Nat Mater 12 (2013) 439

${ }^{129}$ M de la Mata et al, J. Mater. Chem. C 1 (2013) 4300-4312

130 J Arbiol et al, Mater. Today 16 (2013) 213-219

${ }^{131}$ A Fontcuberta i Morral et al, Small, 4, 899-903 (2008)

132 M Heigoldt et al, J. Mater. Chem. 19 (2009) 840-848

${ }_{133}$ M Fickenscher et al, Nano Lett. 13 (2013) 1016-1022

${ }^{134} \mathrm{~J}$ Arbiol et al, Nanoscale 4 (2012) 7517-77524

135 J. Müßener et al., Nanoscale, 10, 5591-5598 (2018)

${ }^{136}$ KA Dick et al, Nano Lett. 12 (2012) 3200-3206

137 V Zannier et al, Nano Lett. 18 (2018) 167-174

${ }^{138} \mathrm{C}$ Zhou et al, Nano Lett. 17 (2017) 7824-7830

${ }^{139} \mathrm{~N}$ Skold et al, Nano Lett. 5 (2005) 1943-1947

$140 \mathrm{H}$ Kindlund et al, Nano Lett. 18 (2018) 5775-5781

${ }^{141} \mathrm{X}$ Lu et al, Chem. Mater. 29 (2017) 9786-9792

142 HY Hui et al, Chem. Mater. 29 (2017) 3397-3402

${ }^{143}$ E Oksenberg, et al, ACS Nano, 11, 6155-6166 (2017)

${ }^{144}$ M Orru et al, Phys. Rev. Mater. 2 (2018) 043404 
${ }^{145} \mathrm{~F}$ Ishizaka et al, Nano Lett. 17 (2017) 1350-1355

${ }^{146}$ HIT Hauge et al, Nano Lett. 15 (2015) 5855-5860

${ }^{147}$ G Zhang et al, Nanotechnology 26 (2015) 115704

148 G Priante at al, Nano Lett. 16 (2016) 1917-1924

${ }^{149}$ AM Smith et al, Nat. Nano 4 (2009) 56

${ }^{150} \mathrm{G}$ Signorello et al, Nano Lett. 13 (2013) 917-924

${ }^{151}$ G Signorello et al, Nano Lett. 17 (2017) 2816-2824

152 DV Beznasyuk et al, Nanotech 28 (2017) 365602

${ }^{153}$ D Scarpellini et al, Nano Lett. 15 (2015) 3677-3683

${ }^{154}$ HY Hui et al, Chem. Mater. 29 (2017) 3397-3402

${ }^{155}$ C Himwas et al, Nanotechnology 28 (2017) 495707

${ }^{156}$ S Vaitiekenas et al., Phys. Rev. Lett., 121, 147701 (2018)

${ }^{157}$ F Krizek et al., Phys. Rev. Mater., 2, 093401 (2018)

${ }^{158} \mathrm{G}$ Tutuncuoglu, et al., Nanoscale, 7, 19453-19460 (2015)

159 M Friedl, Nano Lett., 18, 2666-2671 (2018)

160 F Qian et al, Nano Lett. 4 (2004) 1975-1979

${ }^{161}$ VJ Keast et al, PRB 66 (2002) 125319

${ }^{162}$ S Lazar et al, Ultramicroscopy 96 (2003) 535-546

$163 \mathrm{~J}$ Arbiol et al., Nanotechnology 20 (2009) 145704

${ }^{164}$ PE Batson et al, Phys. Rev. Lett. 57 (1986) 2729-2732

165 D Keller et al, Appl. Phys. Lett. 109 (2016) 153103

${ }^{166}$ LE Luna et al, J Phys. Chem. C 122 (2018) 12047

${ }^{167}$ T Kuykendall et al, Nat. Mater. 6 (2007) 951

168 S Meuret et al, Nano Lett. 18 (2018) 2288-2293

169 B Zhao et al, Nanoscale 10 (2018) 11205-11210

$170 \mathrm{H}-\mathrm{L}$ Chen et al, Nano Lett. 17 (2017) 6667-6675

${ }^{171}$ A Gustafsson, J. Microscopy 262 (2016) 134-141

172 D Lindgren et al, Nanotech 26 (2015) 045705

${ }^{173}$ LF Zagonel et al, Nano Lett. 11 (2011) 568-573

${ }_{174}$ M Kociak, LF Zagonel, Ultramicroscopy 176 (2017) 112-131

${ }^{175}$ A Urban et al, Nano Lett. 15 (2015) 5105-5109

176 M Muller et al, Nano Lett. 16 (2016) 5340-5346

177 S Meuret et al, ACS Photonics 3 (2016) 1157-1163

178 G Sanchez-Santolino et al, ACS Nano 12 (2018) 8875-8881 